\title{
Instabilities in directional solidification under inclined rotation
}

\author{
By C. A. CHUNG ${ }^{1}$ AND FALIN CHEN ${ }^{2}$ \\ ${ }^{1}$ Department of Mechanical Engineering, National Central University, Jungli, Taiwan 320 \\ ${ }^{2}$ Institute of Applied Mechanics, National Taiwan University, Taipei, Taiwan 106 \\ falin@iam.ntu.edu.tw
}

(Received 6 December 2001 and in revised form 16 September 2002)

We investigate the stability of a binary alloy directionally solidifying at a constant rate and rotating with spin and/or precession about an inclined axis. Results show that, prior to the onset of instability, a flow is induced by the inclination and modified by the rotation, having a velocity profile like a spiral Ekman flow. The induced flow moves steadily relative to the system when the system rotates with precession only, while it changes direction periodically when the system rotates with spin (even if precession is included). Based on this flow, the effects of inclined rotation on the stability of the system are examined by linear analyses. We find that there are five mechanisms affecting the stability due to inclined rotation: the reduction of both buoyancy and the rotation vector along the height of the system are stabilizing, the gravity component along the melt/solid interface is destabilizing, and the inclination-induced flow and precession combine to play a stabilizing or a destabilizing role, depending on their relative orientation and amplitude ratio. In general, the morphological mode is slightly stabilized whereas the convective and mixed modes are significantly stabilized. For inclined precession, the instability mode moving aligned with the gravity component along the melt/solid interface is most unstable. For inclined spin, all the stabilityaffecting mechanisms act equally in all directions so that the stability thresholds for the instability modes moving in different directions are equal. For directional solidification applications, the present results suggest that to prevent compositional non-uniformities in the solid, inclined spin is more effective than inclined precession.

\section{Introduction}

During the directional solidification of binary alloys, the solute is either rejected from or incorporated into the solid depending on the alloy system, and a solute boundary layer is formed above the melt/solid interface. In such a circumstance, the interface may become unstable to a cellular structure, resulting in an unwanted compositional inhomogeneity in the solid. This is known as morphological instability, a long-standing concern in, for example, microchip manufacturing technology. For binary solutions, the morphological instability mainly results from the constitutional supercooling of the residual melt above the interface (Rutter \& Chalmers 1953). The perturbed interface encounters the supercooled melt and starts to grow, rendering the interface unstable (Mullins \& Sekerka 1964; Wollkind \& Segal 1970; Ungar \& Brown 1984).

The interface may also lose its planar shape due to convective instability in the melt. This natural convection occurs due to a statically unstable density distribution 
in the residual liquid resulting from either the rejected solute being lighter, or the incorporated solute being heavier, than the solvent. Coriell et al. (1980) investigated the buoyancy-driven instability coupled with the morphological instability and showed that the convective instability is characterized by a wavelength comparable to the thickness of the compositional boundary layer and the wavelength of the morphological mode is much shorter. These two modes are stationary and their neutral curves are connected by the co-called mixed modes, which are oscillatory in nature. Later, Jenkins (1985) conducted a weakly nonlinear analysis of the same problem and indicated the mathematical complexity of the problem. By applying the long-wave approximation, Riley \& Davis $(1990 a, b)$ and Wheeler (1991) were able to investigate the weakly nonlinear behaviour of the problem again and gain a more thorough understanding of the interaction between the convective and morphological instabilities as well as the nature of the bifurcation from the linear critical point. Chen \& Davis (1999) investigated the morphological instability of a solidifying front growing into a pre-existing cellular convective flow, which may occur due to the buoyancy- or inertial-driven instability. They found that the twodimensional convective flow stabilizes two-dimensional disturbances but destabilizes three-dimensional disturbances. When the convective flow is weak, the morphological mode is incommensurate with the flow in terms of disturbance wavelength. When the flow is strong, the instability is forced to fit into the convective flow through the amplitude modulation.

In order to prevent the melt/solid interface becoming corrugated, studying the effect of a shear flow on the morphological instability has been discussed widely (Glicksman, Coriell \& McFadden 1986). Either naturally occurring or artificially imposed shear flows were believed to be able to alter the thresholds of both the morphological and convective instabilities and change the pattern of the interfacial morphology as well. The flow-modified instability has been studied by Delves (1968, 1971) by imposing a Blasius boundary-layer flow above the melt/solid interface, by Coriell et al. (1984) by applying a plane Couette flow parallel with the melt/solid interface, by MacFadden, Coriell \& Alexander (1988) and Brattkus \& Davis (1988) by imposing a plane stagnation flow vertically onto the melt/solid interface, and by Forth \& Wheeler $(1989,1992)$ by imposing an asymptotic suction boundary-layer flow along the melt/solid interface. Forth \& Wheeler (1992) showed that the shear flow serves to decouple the convective and morphological modes, which were originally coupled without shear. Brattkus \& Davis (1988), Davis (1990), and Davis \& Schulze (1996) found a flow-induced morphological instability occurring due to the non-parallel effect when the characteristic wavelength becomes large, as found by Chung \& Chen (2001). These investigations yielded two general conclusions. First, the imposed shear flows have a weak effect on the critical condition of the morphological instability because the morphological instability is characterized by a small wavelength. Second, the imposed shear flows influence both the morphological and convective modes when moving in specific directions. More specifically, a parallel shear flow suppresses the instability modes that have propagation vector with a component parallel with the imposed flow, while it has no effect on the mode propagating in the direction perpendicular to the flow. In other words, the imposed parallel shear flow has only a two-dimensional stabilizing capability, and the two-dimensional instability rolls of an axis aligned with the flow direction occurs preferentially.

To extend the stabilizing effect of shear flow to three dimensions, Schulze \& Davis (1995) investigated a system that oscillates the solid in an elliptical orbit parallel to the interface to generate a time-periodic Stokes-layer-type flow changing direction 
periodically. By selecting an appropriate frequency and amplitude of modulation, they showed that the morphological instability could be significantly reduced. The effect of a similar type of oscillatory Stokes-layer flow has recently been studied by Volfson \& Vinals (2001), who assumed that the amplitude of oscillation of the fluid far from the interface is much smaller than the wavelength of the interfacial corrugation and obtained an analytical form of the dispersion relation between arbitrary wavenumbers and shear flow rates.

Another potential scheme to prevent the melt/solid interface from corrugation is to rotate the system with respect to the vertical axis. Oztekin \& Pearlstein (1992) and Lu $\&$ Chen (1997) showed that vertical rotation in general stabilizes the convective mode by the action of the Coriolis force but has negligible effect on the morphological mode. Oztekin \& Pearlstein also found that the morphological instability is of a small wavelength so that the corresponding flow induced by the morphological instability is virtually perpendicular to the melt/solid interface (i.e. parallel to the rotation axis), rendering the generated Coriolis force insignificant.

In this paper, we propose an alternative scheme, namely inclined rotation with spin and/or precession, which is supposed to contain the stabilizing effects of both shear flow and rotation. This scheme is motivated by the experiment of Sample \& Hellawell (1984) and the analysis of Chung \& Chen (2000). Their work showed that such a scheme may significantly suppress the formation of chimneys within the mushy zone of a solidifying binary alloy because, first, the buoyancy is reduced along the direction of the density gradient due to inclination, and second, an Ekman type of flow moving along the melt/solid interface is induced. This induced flow is driven by the gravity component along the interface and modified by rotation. Unlike previous studies in which shear flows were often artificially imposed, the spiral shear flow occurs naturally in the inclined rotating system. Therefore, this scheme appears more feasible for industrial applications. Note that in the system of Chung \& Chen (2000) there is a mushy layer between the solid and the residual melt, and the instability of the mush was investigated by a linear stability analysis. Owing to the large resistance to the flow in the mush, the corresponding Coriolis force and inclination-induced flow are both very weak. In the present system, in contrast, there is no mushy layer and emphasis is placed on the instabilities of the interfacial morphology and the melt, in which a much larger induced flow and a more influential Coriolis force occur. In addition, in the present system the interaction of the induced shear flow with the precession may also significantly affect the stability, see, for example, Kropp \& Busse (1991), Busse \& Kropp (1992), and Matthews \& Cox (1997). They investigated the interaction between a shear flow and a horizontal rotation vector for buoyancy-driven convection and found that the interaction may be stabilizing or destabilizing depending on the relative orientation and amplitude ratio of the shear flow and rotation vector. More specifically, the rotation alone favours a two-dimensional instability roll with axis aligned with the rotation vector, and the shear flow alone favours a roll aligned with the flow. When both rotation and shear flow co-exist, an oblique roll is preferred, and the critical Rayleigh number may be lower than that without rotation or shear flow. In the present system, because precession has a component parallel to the melt/solid interface, it can interact with the induced flow in a similar way. Thus, a rich and complicated stability behaviour for the present system is expected. Similar rotation systems have also been applied to thin-layer coating problems; see for example Hoffmann \& Busse (2001) and Davalos-Orozco \& Busse (2002).

The paper is organized as follows. The mathematical formulation is given in $\S 2$. The equations and associated boundary conditions are solved analytically for the 


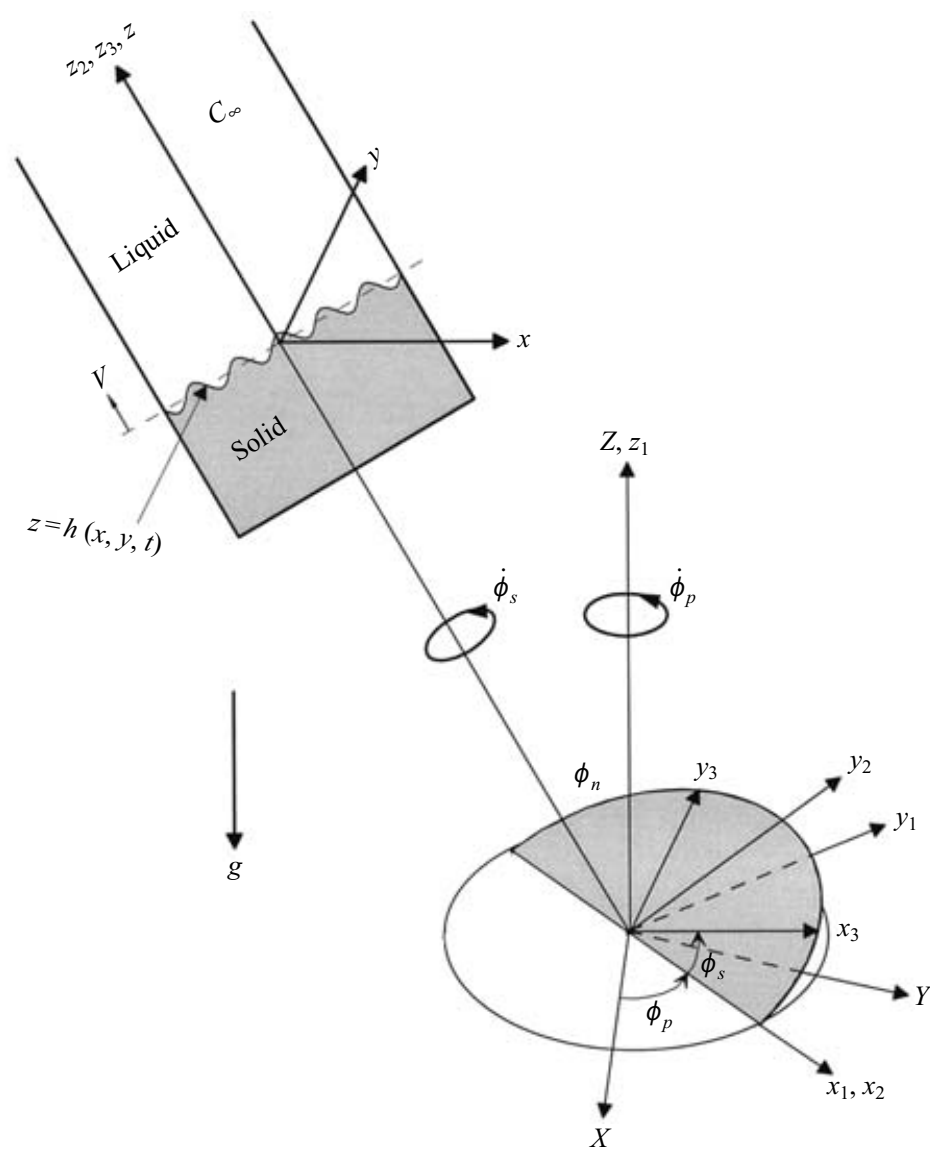

FIGURE 1. A sketch of the inclined rotation system. A binary alloy is directionally solidified from below, and the semi-infinite liquid overlies the solid. The solid is assumed to grow upwards with a constant speed $V$. The frame of reference denoted by oxyz is fixed at the melt/solid interface, rotating with the solid and translating with the interface. $\phi_{n}$ is the inclined angle, $\dot{\phi}_{p}$ is the precession speed, and $\dot{\phi}_{s}$ is the spin speed. The gravity vector points vertically downward.

induced flow in $\S 3$. The linear perturbation equations are derived in $\S 4$. The stability characteristics of the systems under different kinds of inclined rotation are discussed in $\S 5$. Finally, concluding remarks are summarized in $\S 6$.

\section{Problem description and formulation}

The system considered is shown in figure 1, in which a dilute binary alloy of initial temperature $T_{\infty}$ and concentration $C_{\infty}$ is solidified from below, and a solid region forms below the semi-infinite bulk melt. The melt/solid interface, whose position is given by $z=h(x, y, t)$, is assumed to be initially planar and advances into the bulk melt at a constant speed $V$. The system rotates about an inclined axis by spin and/or precession and the angular velocity can be described as

$$
\dot{\boldsymbol{\Phi}}=\left(\dot{\phi}_{p} \sin \phi_{n} \sin \phi_{s}\right) \boldsymbol{e}_{x}+\left(\dot{\phi}_{p} \sin \phi_{n} \cos \phi_{s}\right) \boldsymbol{e}_{y}+\left(\dot{\phi}_{p} \cos \phi_{n}+\dot{\phi}_{s}\right) \boldsymbol{e}_{z},
$$


where $\phi_{n}$ and $\phi_{s}$ are respectively the angles of inclination and spin, and $\dot{\phi}_{p}$ and $\dot{\phi}_{s}$ are the angular velocities of precession and spin. In addition, $\boldsymbol{e}_{x}, \boldsymbol{e}_{y}$ and $\boldsymbol{e}_{z}$ are the unit vectors of the Cartesian coordinate system fixed at the melt/solid interface, rotating with the solid and translating upward at velocity $V$, as denoted by the frame oxyz in figure 1.

With respect to such a reference frame, the governing equations in the fluid region $h<z<\infty$ are the conservation of mass, momentum, solute and heat, respectively

$$
\begin{gathered}
\nabla \cdot \boldsymbol{u}=0 \\
{\left[\frac{\partial}{\partial t}-V \frac{\partial}{\partial z}+\boldsymbol{u} \cdot \nabla\right] \boldsymbol{u}+2 \dot{\boldsymbol{\Phi}} \times \boldsymbol{u}=-\frac{\nabla P}{\rho_{0}}+v \nabla^{2} \boldsymbol{u}+\left(\frac{\rho}{\rho_{0}}-1\right) \boldsymbol{g}} \\
\left(\frac{\partial}{\partial t}-V \frac{\partial}{\partial z}+\boldsymbol{u} \cdot \nabla\right) C=D_{f} \nabla^{2} C \\
\left(\frac{\partial}{\partial t}-V \frac{\partial}{\partial z}+\boldsymbol{u} \cdot \nabla\right) T=\kappa_{f} \nabla^{2} T
\end{gathered}
$$

In these equations, $\boldsymbol{u}$ is the velocity vector $(u, v, w)$ measured relative to the solid, $P=p-\rho_{0} \boldsymbol{g} \cdot \boldsymbol{r}$, where $p$ is the static pressure, $\rho_{0}$ a reference density and $\boldsymbol{r}$ the position vector, and $2 \dot{\boldsymbol{\Phi}} \times \boldsymbol{u}$ is the Coriolis force. Note that in $(2.2 b)$ we have ignored the centrifugal acceleration $\dot{\boldsymbol{\Phi}} \times(\dot{\boldsymbol{\Phi}} \times \boldsymbol{r})$ as well as the tangential acceleration $\ddot{\boldsymbol{\Phi}} \times \boldsymbol{r}$. The relative significance of these two terms compared to gravity can be measured by the ratio $|\dot{\boldsymbol{\Phi}}|^{2} r_{c} / g$, where $r_{c}$ is the characteristic length measured from the axis of rotation, approximately equal to the horizontal dimension of the system. When the speed of spin or precession considered is smaller than 5 r.p.m. and $r_{c}$ is about $25 \mathrm{~cm}$ (Coriell et al. 1984), the ratio is less than 0.01 and so the centrifugal and tangential accelerations are negligible. Finally, $C$ is the concentration, $T$ the temperature, $D_{f}$ the solute diffusivity, $\kappa_{f}$ the thermal diffusivity, $v$ the kinematic viscosity, and $g=-g\left(\sin \phi_{n} \sin \phi_{s}, \sin \phi_{n} \cos \phi_{s}, \cos \phi_{n}\right)$ the gravity vector, depending on both the inclination and spin angles, where $g$ is the gravitational constant. Since the Boussinesq approximation is applied, the fluid density is constant except in the gravity term where the following relation holds:

$$
\rho=\rho_{0}\left(1-\alpha\left(T-T_{m}\right)-\beta C\right)
$$

In this equation $\alpha$ and $\beta$ are respectively the thermal and solute expansion coefficients and $T_{m}$ is the freezing temperature of the pure solvent. In the solid region $z<h$, we ignore the solute diffusion and consider only a heat balance. Thus, the governing equation in the solid layer is

$$
\left(\frac{\partial}{\partial t}-V \frac{\partial}{\partial z}\right) T=\kappa_{s} \nabla^{2} T,
$$

where $\kappa_{s}$ is the thermal diffusivity of the solid phase.

For the boundary conditions, we assume that the fluid in the far field experiences a rigid-body rotation and both the concentration and temperature remain at their original values. Or, equivalently, we assume that the residual melt remains so deep that the influence of the possible deformation of the free surface at the top on the fluid motion near the melt/solid interface can be ignored. Accordingly, at $z \rightarrow \infty$ 
we have

$$
\boldsymbol{u} \rightarrow \mathbf{0}, \quad C \rightarrow C_{\infty}, \quad T \rightarrow T_{\infty} .
$$

At the melt/solid interface $z=h(x, y, t)$, the boundary conditions are

$$
\begin{gathered}
\boldsymbol{u} \times \boldsymbol{n}=\mathbf{0}, \quad \boldsymbol{u} \cdot \boldsymbol{n}=0, \\
C_{+}(1-k)\left(V+\frac{\partial h}{\partial t}\right) \boldsymbol{e}_{z} \cdot \boldsymbol{n}=-D_{f} \frac{\partial C_{+}}{\partial n}, \\
T_{+}=m C_{+}+T_{m}(1-\Gamma \mathscr{K}), \quad T_{+}=T_{-}, \\
L_{h}\left(V+\frac{\partial h}{\partial t}\right) \boldsymbol{e}_{z} \cdot \boldsymbol{n}=k_{s} \frac{\partial T_{-}}{\partial n}-k_{f} \frac{\partial T_{+}}{\partial n},
\end{gathered}
$$

where $\boldsymbol{n}$ is the normal vector to the melt/solid interface directing toward the melt and the subscripts + and - denote respectively quantities just above and below the interface. Equation (2.6a) is the no-slip condition. Equation (2.6b) represents the conservation of mass at the interface in which we have neglected the density difference between the solid and liquid phases. Equation (2.6c) represents the conservation of solute at the interface, where $k=C_{-} / C_{+}$is known as the segregation or partition coefficient. Equation (2.6d) is the thermodynamic equilibrium condition describing the dependence of the freezing temperature of a binary alloy upon its composition, where $m$ is the liquidus slope (assumed to be a constant). In $(2.6 d)$ we have also included the capillary effect (the Gibbs-Thompson effect), where $\Gamma$ is the capillary length and $\mathscr{K}$ is the curvature of the interface (assumed negative for a concave projection into the melt). Equation $(2.6 e)$ is the continuity of temperature across the interface. Finally, equation $(2.6 f)$ is the energy balance at the interface, where $k_{s}$ and $k_{f}$ are the thermal conductivity of the solid and fluid phases respectively, and $L_{h}$ is the latent heat per unit volume of the solid.

The governing equations and boundary conditions are made dimensionless with the solute-field scales: $V$ for velocity, $D_{f} / V$ for length, $D_{f} / V^{2}$ for time, $C_{\infty}$ for concentration, $T_{m}$ for temperature, and $v \rho_{0} V^{2} / D_{f}$ for pressure. When making the temperature dimensionless, we subtracted $T_{m}$ from the dimensional temperature before dividing it with the scale. The dimensionless governing equations in the fluid region are

$$
\begin{gathered}
\nabla \cdot \boldsymbol{u}=0 \\
\frac{1}{S_{c}}\left[\frac{\partial}{\partial t}-\frac{\partial}{\partial z}+\boldsymbol{u} \cdot \nabla\right] u=\nabla^{2} u-\frac{\partial p}{\partial x}+\left(R_{c} C+R_{t} T\right) S_{n} S_{s}(t) \\
+\left[(-1)^{n_{p}} T_{a p}^{1 / 2} C_{n}+(-1)^{n_{s}} T_{a s}^{1 / 2}\right] v-\left[(-1)^{n_{p}} T_{a p}^{1 / 2} S_{n} C_{s}(t)\right] w
\end{gathered}
$$

$$
\begin{aligned}
\frac{1}{S_{c}}\left[\frac{\partial}{\partial t}-\frac{\partial}{\partial z}+\boldsymbol{u} \cdot \nabla\right] v= & \nabla^{2} v-\frac{\partial p}{\partial y}+\left(R_{c} C+R_{t} T\right) S_{n} C_{s}(t) \\
& -\left[(-1)^{n_{p}} T_{a p}^{1 / 2} C_{n}+(-1)^{n_{s}} T_{a s}^{1 / 2}\right] u+\left[(-1)^{n_{p}} T_{a p}^{1 / 2} S_{n} S_{s}(t)\right] w,
\end{aligned}
$$

$$
\begin{aligned}
\frac{1}{S_{c}}\left[\frac{\partial}{\partial t}-\frac{\partial}{\partial z}+\boldsymbol{u} \cdot \nabla\right] w= & \nabla^{2} w-\frac{\partial p}{\partial z}+\left(R_{c} C+R_{t} T\right) C_{n} \\
& +\left[(-1)^{n_{p}} T_{a p}^{1 / 2} S_{n} C_{s}(t)\right] u-\left[(-1)^{n_{p}} T_{a p}^{1 / 2} S_{n} S_{s}(t)\right] v
\end{aligned}
$$




$$
\begin{gathered}
\left(\frac{\partial}{\partial t}-\frac{\partial}{\partial z}+\boldsymbol{u} \cdot \nabla\right) C=\nabla^{2} C, \\
\frac{1}{L_{e}}\left(\frac{\partial}{\partial t}-\frac{\partial}{\partial z}+\boldsymbol{u} \cdot \nabla\right) T=\nabla^{2} T .
\end{gathered}
$$

The dimensionless heat equation in the solid region is

$$
\frac{1}{L_{s}}\left(\frac{\partial}{\partial t}-\frac{\partial}{\partial z}\right) T=\nabla^{2} T .
$$

In these equations, $S_{c} \equiv v / D_{f}$ is the Schmidt number, $L_{e} \equiv \kappa_{f} / D_{f}$ the Lewis number of the fluid phase and $L_{s} \equiv \kappa_{s} / D_{f}$ the Lewis number of the solid phase. In addition, $R_{c}$ and $R_{t}$ are respectively the solutal and thermal Rayleigh numbers defined based on the solute-field length scale $H \equiv D_{f} / V$ :

$$
R_{c} \equiv \frac{g \beta C_{\infty} H^{3}}{\nu D_{f}}, \quad R_{t} \equiv \frac{g \alpha T_{m} H^{3}}{\nu D_{f}},
$$

and $T_{a p}$ and $T_{a s}$, which account for the intensity of rotation, are the Taylor numbers of precession and spin defined respectively as

$$
T_{a p} \equiv\left(\frac{2 H^{2} \dot{\phi}_{p}}{v}\right)^{2}, \quad T_{a s} \equiv\left(\frac{2 H^{2} \dot{\phi}_{s}}{v}\right)^{2}
$$

For simplicity, we have adopted the abbreviations $S_{n} \equiv \sin \phi_{n}, C_{n} \equiv \cos \phi_{n}, S_{s}(t) \equiv$ $\sin (\Omega t)$ and $C_{s}(t) \equiv \cos (\Omega t)$, where $\Omega$ is the dimensionless angular velocity of spin related to $T_{a s}$ by $\Omega \equiv S_{c}(-1)^{n_{s}} T_{a s}^{1 / 2} / 2$. Note that the exponents $n_{p}$ and $n_{s}$ account for the sense of precession and spin respectively: $\dot{\phi}_{p}>0$ corresponds to $n_{p}=0, \dot{\phi}_{s}>0$ to $n_{s}=0, \dot{\phi}_{p}<0$ to $n_{p}=1$, and $\dot{\phi}_{s}<0$ to $n_{s}=1$.

The dimensionless boundary conditions in the far field $z \rightarrow \infty$ are

$$
\boldsymbol{u} \rightarrow \mathbf{0}, \quad C \rightarrow 1, \quad T \rightarrow T_{\infty},
$$

and those at the melt/solid interface $z=h(x, y, t)$ are

$$
\begin{gathered}
\boldsymbol{u} \times \boldsymbol{n}=\mathbf{0}, \quad \boldsymbol{u} \cdot \boldsymbol{n}=0, \\
(k-1) C_{+}\left(1+\frac{\partial h}{\partial t}\right) \boldsymbol{e}_{z} \cdot \boldsymbol{n}=\nabla C_{+} \cdot \boldsymbol{n}, \\
T_{+}=M C_{+}-\mathscr{U} \mathscr{K}, \quad T_{-}=T_{+}, \\
\mathscr{S}\left(1+\frac{\partial h}{\partial t}\right) \boldsymbol{e}_{z} \cdot \boldsymbol{n}=\left(L_{s} \nabla T_{-}-L_{e} \nabla T_{+}\right) \cdot \boldsymbol{n} .
\end{gathered}
$$

Here the parameter $M \equiv m C_{\infty} / T_{m}$ is the dimensionless liquidus slope, $\mathscr{U} \equiv \Gamma V / D_{f}$ is the dimensionless capillary length, and $\mathscr{S} \equiv L / T_{m}\left(\rho c_{p}\right)_{-}$is the Stefan number. Note that in $(2.10 f)$ we have neglected the difference of the specific heats between the solid and liquid phases.

\section{The basic-state: the flow induced by inclination}

To obtain the basic state before the onset of instability occurs, we assume that the horizontal dimension $L$ of the system is much larger than the characteristic length 
scale $H \equiv D_{f} / V$. As a result, one obtains from the scale analysis of the continuity equation that $U / L \approx W / H$, where $U$ and $W$ are the characteristic velocities in the horizontal and vertical directions, respectively. We assume also $H \ll L$ and obtain $W \ll U$, or the $z$-component velocity is negligible. Note that the assumption $H \ll L$ also implies that the diffusion in the horizontal direction is negligibly small compared to that in the vertical direction, namely $\partial^{2} / \partial x^{2} \ll \partial^{2} / \partial z^{2}$ and $\partial^{2} / \partial y^{2} \ll \partial^{2} / \partial z^{2}$.

We assume further that the advection terms in $(2.7 b-d)$ are also negligible in the basic state. This assumption is valid under different rotation situations. First, for the system rotating by inclined precession, because the precession speed $\dot{\phi}_{p}$ is independent of time, the time scale of solidification $D_{f} / V^{2}$ dominates the basic state before any small-scale fluid motion occurs due to the convective or morphological instability. The unsteady term $\partial / \partial t$, if it exists, would be of the same order of magnitude as the term measuring solidification (the second term on the left-hand side of $(2.7 b-d))$. Therefore, to neglect the advection term, the advection term must be much smaller than the solidification term, or $L / H \gg U / V$. On the other hand, for the system rotating by inclined spin with or without precession, the advection term is also negligible compared to the solidification term if the condition $L / H \gg U / V$ holds. Moreover, the advection term can also be ignored due to another factor. Because the spin speed $\dot{\phi}_{s}$ depends on time and so do the gravity and Coriolis terms in the equations, physically both the gravity vector and the induced flow change direction periodically with the angular frequency of spin, so that the time scale of spin becomes dominant when the corresponding Strouhal number is sufficiently large, or $\dot{\phi}_{s} L / U \gg 1$. The advection term therefore can be neglected compared to the unsteady term due to spin. For the parameter ranges corresponding to the lead-tin alloy considered in this paper, it is much easier to meet the condition of a large Strouhal number than to meet $L / H \gg U / V$, which will be discussed in more detail at the end of the section.

Given the assumptions above and owing to the inclination (see the third term on the right-hand side of $(2.7 b, c))$, there is a basic flow induced by the gravity component in the $(x, y)$-plane (or along the melt/solid interface). Since the induced flow turns out to be parallel to the $(x, y)$-plane, the basic-state temperature and concentration are not affected, remaining similar to those found in previous studies (for example, McFadden et al. 1988; Davis 1990; Forth \& Wheeler 1989, 1992). The basic-state solutions are given in the following. The planar interface is located at

$$
h_{b}=0 \text {. }
$$

The solute and temperature distributions in the bulk melt region are

$$
\begin{aligned}
C_{b} & =1-G_{c} \mathrm{e}^{-z}, \quad z>0, \\
T_{b} & =T_{\infty}-L_{e} G_{l} \mathrm{e}^{-z / L_{e}}, \quad z>0,
\end{aligned}
$$

where the local gradients of concentration and temperature are defined respectively as

$$
\begin{aligned}
G_{c} & =\frac{k-1}{k}, \\
G_{l} & =\frac{T_{\infty}-M / k}{L_{e}} .
\end{aligned}
$$


In the solid region, the concentration is uniform while the temperature increases exponentially with height, namely

$$
\begin{gathered}
C_{b}=1, \quad z<0 \\
T_{b}=T_{\infty}+\mathscr{S}-\left(\mathscr{S}+L_{e} G_{l}\right) \mathrm{e}^{-z / L_{s}}, \quad z<0 .
\end{gathered}
$$

For the basic flow, the $z$-component is zero as a result of the assumption made at the beginning of the section, i.e.

$$
w_{b}=0 \text {. }
$$

The other two velocity components are obtained by the following procedure. Since the fluid in the far field is assumed to move as a rigid body with the solid, a consideration of $(2.9 a-c)$ suggests the basic-state pressure is of the form

$$
p_{b}=\bar{p}_{b}(z, t)+\left(R_{c}+R_{t} T_{\infty}\right)\left[x S_{n} S_{s}(t)+y S_{n} C_{s}(t)+z C_{n}\right],
$$

where the reduced pressure $\bar{p}_{b}$ is obtained by substituting (3.9) into (2.7d), yielding

$$
\frac{\partial \bar{p}_{b}}{\partial z}=\left[(-1)^{n_{p}} T_{a p}^{1 / 12} S_{n} C_{s}(t)\right] u_{b}-\left[(-1)^{n_{p}} T_{a p}^{1 / 2} S_{n} S_{s}(t)\right] v_{b}+\left[R_{c}\left(C_{b}-1\right)+R_{t}\left(T_{b}-T_{\infty}\right)\right] C_{n} .
$$

Note that the $z$-component of the basic-state pressure gradient balances the $z$ component of the fluid weight in the whole melt and the $x$-and $y$-components of the pressure gradient also balance the corresponding components of the fluid weight in the far field. Consequently, the melt is motionless in the $z$-direction, i.e. $w_{b}=0$ and quiescent in the far field. The pressure gradient cannot balance the fluid weight along the $(x, y)$-plane, which induces a basic flow along the $(x, y)$-plane, which is then modified by the Coriolis force due to rotation. The basic velocity components $u_{b}$ and $v_{b}$ are obtained by substituting (3.8) and (3.9) into $(2.7 b, c)$, yielding

$$
u_{b}+\mathrm{i} v_{b}=U_{b}(z) \mathrm{e}^{\mathrm{i} \phi_{g}},
$$

in which $\mathrm{i}=\sqrt{-1}$ and $U_{b}(z)$ is the amplitude of the basic-state velocity

$$
\begin{aligned}
U_{b}(z)= & -\frac{S_{n} R_{c} G_{c}}{\Delta_{c}} \exp \left(\mathrm{i} \phi_{c}-z\right)-\frac{S_{n} R_{t} L_{e} G_{l}}{\Delta_{t}} \exp \left(\mathrm{i} \phi_{t}-z / L_{e}\right) \\
& +\left[\frac{S_{n} R_{c} G_{c}}{\Delta_{c}} \mathrm{e}^{i \phi_{c}}+\frac{S_{n} R_{t} L_{e} G_{l}}{\Delta_{t}} \mathrm{e}^{i \phi_{t}}\right] \exp \left(-\mathrm{i} b z-z / d_{E}\right)
\end{aligned}
$$

and $\phi_{g}$ is the phase angle of the gravity vector

$$
\phi_{g}=-\left(\Omega t+\frac{1}{2} \pi\right) .
$$

The remaining parameters in $(3.12 a)$ are defined as follows:

$$
\begin{gathered}
d_{E}=1 /\left(\frac{1}{2 S_{c}}+a\right), \\
a=\left[\left(\frac{1}{S_{c}^{2}}+\sqrt{\frac{1}{S_{c}^{4}}+16 T_{e}^{2}}\right) / 8\right]^{1 / 2}, \quad b=\frac{T_{e}}{\left|T_{e}\right|}\left[\left(-\frac{1}{S_{c}^{2}}+\sqrt{\frac{1}{S_{c}^{4}}+16 T_{e}^{2}}\right) / 8\right]^{1 / 2},
\end{gathered}
$$

$$
\Delta_{c}=\left(\left(1-1 / S_{c}\right)^{2}+T_{e}^{2}\right)^{1 / 2}
$$




$$
\begin{gathered}
\cos \phi_{c}=\frac{1-1 / S_{c}}{\Delta_{c}}, \quad \sin \phi_{c}=\frac{T_{e}}{\Delta_{c}}, \\
\Delta_{t}=\left(\left(\left(1 / L_{e}-1 / S_{c}\right) / L_{e}\right)^{2}+T_{e}^{2}\right)^{1 / 2}, \\
\cos \phi_{t}=\frac{\left(1 / L_{e}-1 / S_{c}\right) / L_{e}}{\Delta_{t}}, \quad \sin \phi_{t}=\frac{T_{e}}{\Delta_{t}},
\end{gathered}
$$

in which $T_{e}$ is the effective Taylor number defined as

$$
T_{e} \equiv(-1)^{n_{p}} T_{a p}^{1 / 2} C_{n}+\frac{(-1)^{n_{s}}}{2} T_{a s}^{1 / 2} .
$$

Through the definition of this parameter the combined effect of precession and spin, which may have the same or opposite direction of rotation, can be absorbed into a single physical term. Physically, a larger $T_{e}$ accounts simply for a higher rotation speed and vice versa. With this parameter, the effect of inclined rotation by precession and/or spin on the amplitude of induced velocity can be understood in a more straightforward sense.

Equations (3.11) and (3.12) indicate that the induced flow increases in amplitude with the inclination angle and varies in direction periodically with time at a frequency equal to the spin angular speed $\Omega$. Furthermore, the induced velocity changes with height and is composed of three regions with different length scales. The first region (varying with $e^{-z}$ ) has the length scale of the solute boundary layer and will be termed the solutal-layer flow. The second region (varying with $\mathrm{e}^{-z / L_{e}}$ ) has the length scale of the thermal boundary layer and will be termed the thermal-layer flow. The third region (varying with $\mathrm{e}^{-z / d_{E}}$ ) has the length scale of Ekman-layer spiral flow and changes direction with a period $2 \pi / b$, and will be termed the Ekman-layer flow. A larger speed of rotation (i.e. a larger $T_{e}$ ) results in a larger value of $b$ and thus a shorter turnover period of Ekman-layer flow. Note that $d_{E} \rightarrow S_{c}$ and $b \rightarrow 0$ when $T_{e} \rightarrow 0$, implying that the Ekman-layer flow will be reduced to a boundary-layer flow under asymptotic suction with the length scale $S_{c}$. Note that it can be seen from (3.13) that the induced velocity is still of finite value even if $T_{e} \rightarrow 0$.

To illustrate these three regions of the induced flow, we display in figure 2 their distributions with height for $T_{e}=10$. For all the computations in this paper, the values of the parameter used are listed in table 1, which correspond to the lead-tin alloy considered by Coriell et al. (1980). In figure 2(a), one can see that the velocities of both the solutal-layer and the Ekman-layer flows decrease exponentially with height. The magnitude of the thermal-layer flow velocity remains virtually constant near the melt/solid interface because the large Lewis number considered has resulted in a deep thermal boundary layer. It is evident from figure $2(b)$ that both the solutal-layer and thermal-layer flows do not change direction whereas the Ekman-layer flow changes direction periodically with height. Note that in figure $2(b)$ we have adopted $\phi_{g}$, the orientation of the gravity component in the $(x, y)$-plane, as the reference angle to measure the flow direction. To illustrate the spiral structure of the flow, we show the velocity vector of the induced flow in figure 2(c). The induced flow in the far field remains in the $(x, z)$-plane, while near the interface it has a component in the $y$ direction. The direction of the thermal-layer flow on the interface is virtually opposite to that of the Ekman-layer flow, and the combination of these two flows results in a flow of small magnitude, which is then diminished by the solutal-layer flow, leading 
(a)

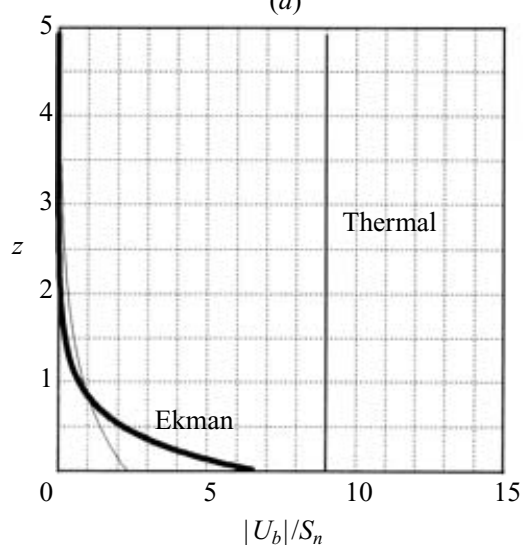

(b)

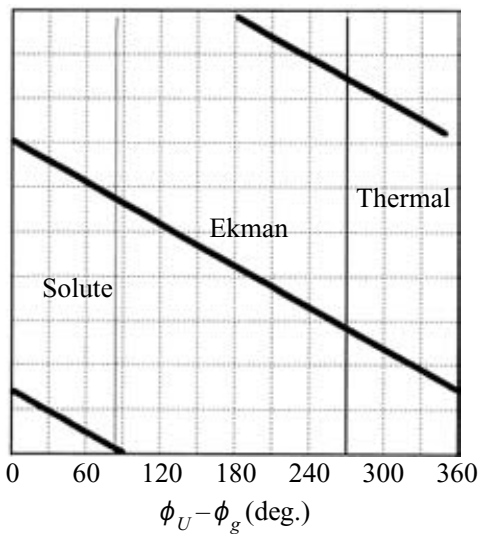

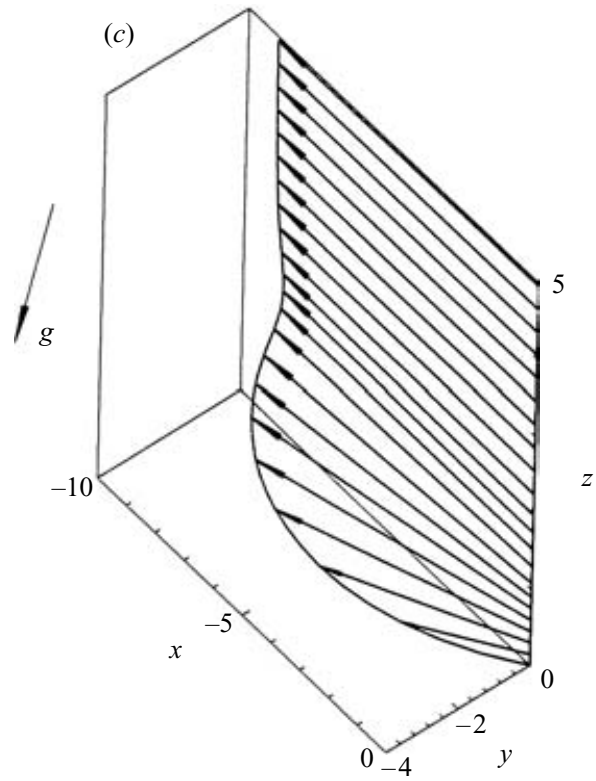

FIGURE 2. The velocity distributions of the three components of the induced basic flow at $T_{e}=10$. (a) The amplitude. (b) The phase angle relative to the gravity component in the $(x, y)$-plane. Note that the Ekman-layer flow varies periodically with height with a period of $2 \pi / b$. (c) The velocity vectors of the induced spiral flow.

$\begin{array}{lc}S_{c} & 81 \\ L_{e} & 3600 \\ L_{s} & 6700 \\ R_{c} & 10 \\ R_{t} & 250 \\ \mathscr{U}^{*} & 6.131 \times 10^{-4} \\ k & 0.3 \\ G_{l} & 10^{-4} \\ \mathscr{S} & 0.29\end{array}$

TABLE 1. The physical parameters used in the numerical calculations corresponding to a lead-tin alloy. 

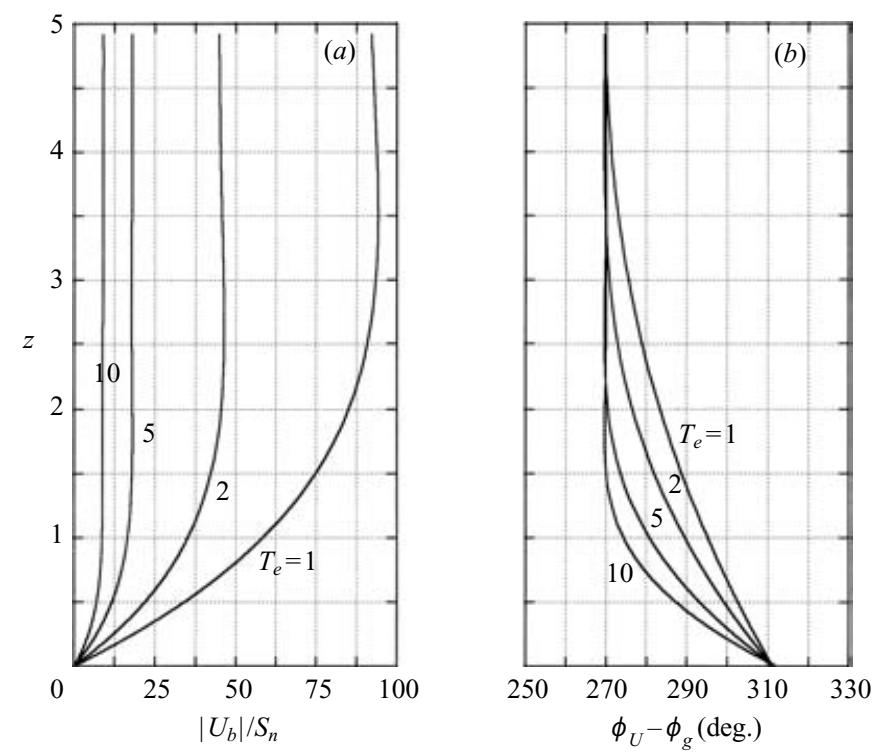

FIGURE 3. (a) The amplitudes of the induced flow velocities for different $T_{e}$. (b) The variation of the phase angle relative to the gravity component in the $(x, y)$-plane for different $T_{e}$.

to the no-slip condition at the interface. At $z \approx 1.4$, however, the Ekman-layer flow and the thermal-layer flow are in the same direction, resulting in the largest amplitude of the induced flow velocity.

In figure 3, the induced flow is illustrated for different values of $T_{e}$. Results show that the velocity decreases as $T_{e}$ increases, indicating that the induced flow is inhibited by a higher spin or precession speed under the action of the Coriolis force. On the other hand, the velocity increases with the inclination angle $\phi_{n}$, implying that the gravity component in the $(x, y)$-plane is a main factor driving the flow. The flow direction changes with height; the relative phase angle $\phi_{U}-\phi_{g}$ decreases from approximately $310^{\circ}$ to $270^{\circ}$ and ultimately remains the same in the rest of the fluid region, where the flow leads gravity by $90^{\circ}$ (i.e. $\phi_{U}-\phi_{g}=270^{\circ}$ ) and this feature applies to all the cases considered in the present paper.

Before proceeding to the stability analysis, it is worth examining the validity of the basic-state solution, which was derived based on the assumption that the system has a much larger horizontal dimension $L$ than the length scale of solidification $H=D_{f} / V$. Consequently, as stated at the beginning of this section, in order to neglect both the horizontal diffusion and the vertical component of the basic velocity, it is necessary to have $L / H \gg 1$. And in order to ignore the advection term of the basic flow, another requirement is $L / H \gg U / V$ when the system rotates with precession, and the smaller of $L / H \gg U / V$ and $\dot{\phi}_{s} L / U \gg 1$ when the system rotates with spin with/without precession. Note that the last condition can be rewritten as $L / H \gg U / \dot{\phi}_{s} H=2 U / S_{c} T_{a s}^{1 / 2} V$, corresponding to the Strouhal number. Given the parameter values in table 1 , we find that the magnitude of the basic flow is ultimately dominated by the thermal-layer flow, i.e. $U / V \propto S_{n} R_{t} L_{e} G_{l} / \Delta_{t}$, which has a value about 5 when the rotation speed (in terms of $T_{e}$ ) is 5 r.p.m. and the inclination angle is $30^{\circ}$ (see figure 3). Therefore, to ignore the advection term for the precession case, the requirement is $L / H \gg 5$, which is only a little more rigorous than the condition $L / H \gg 1$ needed for ignoring both the horizontal diffusion and vertical velocity, but 
is still practical physically because $H$ is generally of magnitude about $1 \mathrm{~cm}$. On the other hand, when spin is imposed at 5 r.p.m. and $30^{\circ}$, a large Strouhal number $L / H \gg U / \dot{\phi}_{s} H=2 U / S_{c} T_{a s}^{1 / 2} V$ yields approximately $L / H \gg 1 / S_{c}$. Since the Schmidt number is generally quite large $\left(S_{c}=81\right.$ in the present paper), this condition will be much easier to satisfy than $L / H \gg U / V \approx 5$. Note that the condition $L / H \gg 1 / S_{c}$ is even less rigorous than $L / H \gg 1$ and so the aspect ratio adequate for the spin case is eventually determined by $L / H \gg 1$. In summary, for the case where the system rotates with precession only, the requirement for the validity of the basic flow is $L / H \gg U / V$, whereas for the case where the system rotates with spin with/without precession, it is $L / H \gg 1$. Both are practical physically.

\section{Linear stability analysis}

We investigate the linear stability of the basic state by focusing particularly on the effects of inclined rotation. To simplify the analysis, the frozen temperature assumption (Langer 1980; Davis 1990; Forth \& Wheeler 1989, 1992) is employed, in which the temperature is fixed at its basic-state value. We introduce small perturbations together with the basic state into $(2.7 a-e)$ and then neglect the products of small quantities to obtain the linear perturbation equations. After eliminating the pressure and the velocities in both the $x$ - and $y$-directions, the linear perturbation equations in the fluid region are

$$
\begin{aligned}
& \left(\frac{\partial}{\partial t}-\frac{\partial}{\partial z}+u_{b} \frac{\partial}{\partial x}+v_{b} \frac{\partial}{\partial y}-\nabla^{2}\right) C=-C_{b}^{\prime} w, \\
& \frac{1}{S_{c}}\left[\left(\frac{\partial}{\partial t}-\frac{\partial}{\partial z}+u_{b} \frac{\partial}{\partial x}+v_{b} \frac{\partial}{\partial y}-S_{c} \nabla^{2}\right) \nabla^{2}-\left(u_{b}^{\prime \prime} \frac{\partial}{\partial x}+v_{b}^{\prime \prime} \frac{\partial}{\partial y}\right)\right] w \\
& +\left\{S_{n}(-1)^{n_{p}} T_{a p}^{1 / 2}\left[S_{s}(t) \frac{\partial}{\partial x}+C_{s}(t) \frac{\partial}{\partial y}\right]+\left[C_{n}(-1)^{n_{p}} T_{a p}^{1 / 2}+(-1)^{n_{s}} T_{a s}^{1 / 2}\right] \frac{\partial}{\partial z}\right\} \zeta \\
& =R_{c}\left\{C_{n} \nabla_{H}^{2}-S_{n}\left[S_{s}(t) \frac{\partial}{\partial x}+C_{s}(t) \frac{\partial}{\partial y}\right] \frac{\partial}{\partial z}\right\} C, \\
& \frac{1}{S_{c}}\left(\frac{\partial}{\partial t}-\frac{\partial}{\partial z}+u_{b} \frac{\partial}{\partial x}+v_{b} \frac{\partial}{\partial y}-S_{c} \nabla^{2}\right) \zeta \\
& +\left\{\frac{1}{S_{c}}\left(v_{b}^{\prime} \frac{\partial}{\partial x}-u_{b}^{\prime} \frac{\partial}{\partial y}\right)-S_{n}(-1)^{n_{p}} T_{a p}^{1 / 2}\left[S_{s}(t) \frac{\partial}{\partial x}+C_{s}(t) \frac{\partial}{\partial y}\right]\right\} w \\
& -\left[C_{n}(-1)^{n_{p}} T_{a p}^{1 / 2}+(-1)^{n_{s}} T_{a s}^{1 / 2}\right] \frac{\partial w}{\partial z}=S_{n} R_{c}\left[C_{s}(t) \frac{\partial}{\partial x}-S_{s}(t) \frac{\partial}{\partial y}\right] C .
\end{aligned}
$$

The associated boundary conditions at $z \rightarrow \infty$ are

$$
C \rightarrow 0, \quad w \rightarrow 0, \quad \frac{\partial w}{\partial z} \rightarrow 0, \quad \zeta \rightarrow 0 .
$$

The boundary conditions at the perturbed melt/solid interface are transformed to the fixed position $z=0$, yielding

$$
\frac{\partial C}{\partial z}-(k-1) C=G_{c}\left(\frac{\partial h}{\partial t}+k h\right),
$$




$$
\begin{gathered}
\frac{C}{G_{c}}=\left(\frac{1}{S_{k}}-1-\mathscr{U}^{*} \nabla_{H}^{2}\right) h, \\
w=0, \\
\frac{\partial w}{\partial z}=u_{b}^{\prime} \frac{\partial h}{\partial x}+v_{b}^{\prime} \frac{\partial h}{\partial y}, \\
\zeta=u_{b}^{\prime} \frac{\partial h}{\partial y}-v_{b}^{\prime} \frac{\partial h}{\partial x} .
\end{gathered}
$$

In these equations, $C$ is the concentration, $w$ and $\zeta$ are respectively the velocity and vorticity in the $z$-direction, and $h=h(x, y, t)$ is the interface perturbation, (all of these are small perturbation quantities), and $\nabla_{H}^{2}=\partial^{2} / \partial x^{2}+\partial^{2} / \partial y^{2}$ is the two-dimensional Laplace operator. In $(4.3 b)$, there are two new dimensionless parameters: the modified capillary parameter $\mathscr{U}^{*}$ and the Sekerka number $S_{k}$, measuring the intensity of the supercooling effect at the interface. They are defined as

$$
\mathscr{U}^{*}=\mathscr{U} / M G_{c}, \quad S_{k}=M G_{c} / G_{l} .
$$

The governing equations (4.1) subject to the boundary conditions (4.2) and (4.3) need to be solved by different approaches depending on whether spin is applied. For the case where the system rotates with precession only, the basic flow is stationary; for the case where the system rotates with spin with/without precession, the basic flow is periodic in time. The details of these two approaches are given in the following subsections.

\subsection{Linear stability analysis: rotation by inclined precession}

When the system rotates with inclined precession, the coefficients of (4.1) are functions of $z$ only. We can apply the normal-mode expansions to the disturbances by employing

$$
\left[\begin{array}{l}
C(x, y, z, t) \\
w(x, y, z, t) \\
\zeta(x, y, z, t) \\
h(x, y, t)
\end{array}\right]=\left[\begin{array}{l}
\hat{C}(z) \\
\hat{w}(z) \\
\hat{\zeta}(z) \\
\hat{h}
\end{array}\right] \exp \left[\left(\omega_{r}+\mathrm{i} \omega_{i}\right) t+\mathrm{i}\left(\alpha_{x} x+\alpha_{y} y\right)\right]+\mathrm{c.c} .
$$

where $\omega_{r}$ and $\omega_{i}$ are the growth rate and the oscillatory frequency of the disturbances respectively, and $\alpha_{x}$ and $\alpha_{y}$ are the wavenumbers in the $x$-and $y$-directions respectively. After substituting these expansions into (4.1), we transform the governing equations into the ordinary differential equations

$$
\begin{gathered}
{\left[\mathrm{D}^{2}+\mathrm{D}-\left(\omega_{r}+\alpha^{2}\right)-\mathrm{i}\left(\omega_{i}+\alpha_{x} u_{b}+\alpha_{y} v_{b}\right)\right] \hat{C}=C_{b}^{\prime} \hat{w}} \\
{\left[\mathrm{D}^{2}+\frac{\mathrm{D}}{S_{c}}-\left(\frac{\omega_{r}}{S_{c}}+\alpha^{2}\right)-\frac{\mathrm{i}}{S_{c}}\left(\omega_{i}+\alpha_{x} u_{b}+\alpha_{y} v_{b}\right)\right]\left(\mathrm{D}^{2}-\alpha^{2}\right) \hat{w}+\frac{\mathrm{i}}{S_{c}}\left(\alpha_{x} u_{b}^{\prime \prime}+\alpha_{y} v_{b}^{\prime \prime}\right) \hat{w}} \\
-T_{a p}^{1 / 2}\left(C_{n} \mathrm{D}+\mathrm{i} S_{n} \alpha_{y}\right) \hat{\zeta}=R_{c}\left(C_{n} \alpha^{2}+i S_{n} \alpha_{y} \mathrm{D}\right) \hat{C} \\
{\left[\mathrm{D}^{2}+\frac{\mathrm{D}}{S_{c}}-\left(\frac{\omega_{r}}{S_{c}}+\alpha^{2}\right)-\frac{\mathrm{i}}{S_{c}}\left(\omega_{i}+\alpha_{x} u_{b}+\alpha_{y} v_{b}\right)\right] \hat{\zeta}} \\
+\left[\frac{\mathrm{i}}{S_{c}}\left(\alpha_{y} u_{b}^{\prime}-\alpha_{x} v_{b}^{\prime}\right)+T_{a p}^{1 / 2}\left(C_{n} \mathrm{D}+\mathrm{i} S_{n} \alpha_{y}\right)\right] \hat{w}=-\mathrm{i} S_{n} \alpha_{x} R_{c} \hat{C},
\end{gathered}
$$


in which the operator $\mathrm{D}$ denotes differentiation with respect to $z$. The associated boundary conditions at $z=0$ become

$$
\begin{gathered}
\mathrm{D} \hat{C}-\left[\frac{(k-1)(F+1)+1+\omega_{r}}{F}+\mathrm{i} \frac{\omega_{i}}{F}\right] \hat{C}=0, \\
\hat{w}=0, \\
\mathrm{D} \hat{w}-\mathrm{i}\left(\frac{\alpha_{x} u_{b}^{\prime}+\alpha_{y} v_{b}^{\prime}}{F G_{c}}\right) \hat{C}=0, \\
\zeta-\mathrm{i}\left(\frac{\alpha_{y} u_{b}^{\prime}-\alpha_{x} v_{b}^{\prime}}{F G_{c}}\right) \hat{C}=0,
\end{gathered}
$$

where $F$ is a function defined as

$$
F=\frac{1}{S_{k}}-1+\mathscr{U}^{*} \alpha^{2}
$$

in which $\alpha=\sqrt{\alpha_{x}^{2}+\alpha_{y}^{2}}$ is the wavenumber. The boundary conditions at $z \rightarrow \infty$ are

$$
\hat{C} \rightarrow 0, \quad \hat{w} \rightarrow 0, \quad \mathrm{D} \hat{w} \rightarrow 0, \quad \hat{\zeta} \rightarrow 0 .
$$

Since both $\alpha_{x}$ and $\alpha_{y}$ appear in both (4.6) and $(4.7 c, d)$, these equations are not symmetric in the $x$ - and $y$-directions. This asymmetry arises from the action of three factors. The first is the gravity component in the $(x, y)$-plane corresponding to the terms containing $S_{n} R_{c}$ on the right-hand side of $(4.6 b, c)$. The second is the precession component in the $(x, y)$-plane corresponding to the terms containing $S_{n} T_{a p}^{1 / 2}$ in $(4.6 b, c)$. The last is the basic-state spiral flow, leading to the appearance of $\alpha_{x}$ and $\alpha_{y}$ together with the basic-state velocities $u_{b}$ and $v_{b}$ in (4.6) and (4.7c, $\left.d\right)$. Since $\alpha_{x}=\alpha \cos \left(\phi_{\alpha}\right)$ and $\alpha_{y}=\alpha \sin \left(\phi_{\alpha}\right)$, where $\phi_{\alpha}$ is the angle between the propagation direction of the instability mode and the $x$-axis, one needs to consider $-180^{\circ} \leqslant \phi_{\alpha} \leqslant 180^{\circ}$ for a complete analysis. But owing to the complex conjugate of the expansions in (4.5), one need only take $-90^{\circ} \leqslant \phi_{\alpha} \leqslant 90^{\circ}$ into account. Equations (4.6) to (4.8) constitute a complex eigenvalue problem, which can be solved by a shooting technique (see Chung \& Chen 2000). In numerical calculations, the truncation height of the system is set to a finite value (Coriell et al. 1980, 1984), which depends on the value of $\alpha$. When $\alpha=O(5)$, choosing the truncation height equal to 20 gives a deviation of $S_{k}$ less than $O\left(10^{-4}\right)$. When $\alpha=O(0.5)$, the truncation height needs to be about 100 to retain the same accuracy of $S_{k}$.

\subsection{Linear stability analysis: rotation by inclined spin with/without precession}

When inclined spin is imposed, the basic flow becomes a periodic function of time, varying by a frequency equal to the spin angular velocity $\Omega$. Consequently, (4.1) contains time-dependent coefficients and needs to be handled with the Floquet theory by expanding the time-dependent variable into a complex Fourier series in time. We found when applying normal-mode expansions that $\alpha_{x}$ and $\alpha_{y}$ always appear with the following forms:

$$
\begin{gathered}
\alpha_{x} \sin (\Omega t)+\alpha_{y} \cos (\Omega t)=\alpha \sin \left(\Omega t+\phi_{\alpha}\right)=\alpha \sin \left(\Omega t^{\prime}\right), \\
\alpha_{y} \sin (\Omega t)-\alpha_{x} \cos (\Omega t)=-\alpha \cos \left(\Omega t+\phi_{\alpha}\right)=-\alpha \cos \left(\Omega t^{\prime}\right),
\end{gathered}
$$

where $t^{\prime}$, defined as

$$
t^{\prime}=t+\frac{\phi_{\alpha}}{\Omega},
$$


is the time shift for the instability mode propagating in the direction of $\phi_{\alpha}$. With this new variable, $\phi_{\alpha}$ is removed from the equations after the normal-mode expansion is applied. This implies that the disturbances propagating in different directions have the same stability characteristics; the only difference between them is the temporal phase $\Delta \phi_{\alpha} / \Omega$. Physically, the gravity vector, the precession vector and the basic flow change their directions synchronously with the spin. As a result, all the disturbances will sense the effects of these three mechanisms within a certain period of time, and the symmetric situation in the $(x, y)$-plane therefore holds; accordingly, this will be called 'stability symmetry in the $(x, y)$-plane' hereafter. Note that these three mechanisms have caused the asymmetry in the case of inclined precession mentioned in the previous subsection.

After applying the Floquet theory and the normal-mode expansions

$$
\left[\begin{array}{l}
C(x, y, z, t) \\
w(x, y, z, t) \\
\zeta(x, y, z, t) \\
h(x, y, t)
\end{array}\right]=\sum_{|l| \leqslant L_{n}}\left[\begin{array}{l}
C_{l}(z) \\
w_{l}(z) \\
\zeta_{l}(z) \\
h_{l}
\end{array}\right] \exp \left[\left(\omega_{r}+\mathrm{i} \omega_{i}+\mathrm{i} l \Omega\right) t^{\prime}\right] \exp \left(\mathrm{i} \alpha_{x} x+\mathrm{i} \alpha_{y} y\right)+\text { c.c., }
$$

(4.1) are transformed into the ordinary differential equations

$$
\begin{aligned}
& {\left[\mathrm{D}^{2}+\mathrm{D}-\left(\omega_{r}+\alpha^{2}\right)-\mathrm{i}\left(\omega_{i}+l \Omega\right)\right] C_{l}-C_{b}^{\prime} w_{l}+f_{11}\left(C_{l-1}-C_{l+1}\right)+\mathrm{i} f_{12}\left(C_{l-1}+C_{l+1}\right)=0,} \\
& {\left[\begin{array}{l}
\mathrm{D}^{4} \\
+
\end{array} \frac{\mathrm{D}^{3}}{S_{c}}-\left(\frac{\omega_{r}}{S_{c}}+2 \alpha^{2}\right) \mathrm{D}^{2}-\frac{\alpha^{2}}{S_{c}} \mathrm{D}+\alpha^{2}\left(\alpha^{2}+\frac{\omega_{r}}{S_{c}}\right)-\frac{\mathrm{i}}{S_{c}}\left(\omega_{i}+l \Omega\right)\left(\mathrm{D}^{2}-\alpha^{2}\right)\right] w_{l}} \\
& +\frac{f_{11}}{S_{c}}\left(\mathrm{D}^{2}-\alpha^{2}\right)\left(w_{l-1}-w_{l+1}\right)+\mathrm{i} \frac{f_{12}}{S_{c}}\left(\mathrm{D}^{2}-\alpha^{2}\right)\left(w_{l-1}+w_{l+1}\right)+\frac{f_{21}}{S_{c}}\left(w_{l-1}-w_{l+1}\right) \\
& +\mathrm{i} \frac{f_{22}}{S_{c}}\left(w_{l-1}+w_{l+1}\right)-\left[C_{n}(-1)^{n_{p}} T_{a p}^{1 / 2}+(-1)^{n_{s}} T_{a s}^{1 / 2}\right] \mathrm{D} \zeta_{l} \\
& -\frac{S_{n} \alpha(-1)^{n_{p}} T_{a p}^{1 / 2}}{2}\left(\zeta_{l-1}-\zeta_{l+1}\right)=C_{n} R_{c} \alpha^{2} C_{l}+\frac{S_{n} \alpha}{2} \mathrm{D}\left(C_{l-1}-C_{l+1}\right), \\
& \quad\left[\mathrm{D}^{2}+\frac{\mathrm{D}}{S_{c}}-\left(\frac{\omega_{r}}{S_{c}}+\alpha^{2}\right)-\frac{\mathrm{i}}{S_{c}}\left(\omega_{i}+l \Omega\right)\right] \zeta_{l}+\frac{f_{11}}{S_{c}}\left(\zeta_{l-1}-\zeta_{l+1}\right)+\mathrm{i} \frac{f_{12}}{S_{c}}\left(\zeta_{l-1}+\zeta_{l+1}\right) \\
& \quad+\left[C_{n}(-1)^{n_{n}} T_{a p}^{1 / 2}+(-1)^{n_{s}} T_{a s}^{1 / 2}\right] \mathrm{D} w_{l}+\frac{S_{n} \alpha(-1)^{n_{p}} T_{a p}^{1 / 2}}{2}\left(w_{l-1}-w_{l+1}\right) \\
& \quad+\mathrm{i} \frac{S_{n} R_{c} \alpha}{2}\left(C_{l-1}+C_{l+1}\right)+\frac{f_{31}}{S_{c}}\left(w_{l-1}-w_{l+1}\right)+\mathrm{i} \frac{f_{32}}{S_{c}}\left(w_{l-1}+w_{l+1}\right)=0 .
\end{aligned}
$$

The associated boundary conditions at $z=0$ are transformed into

$$
\begin{gathered}
\mathrm{D} C_{l}-\left[\frac{(k-1)(F+1)+1+\omega_{r}}{F}+\mathrm{i} \frac{\omega_{i}+l \Omega}{F}\right] C_{l}=0, \\
w_{l}=0, \\
\mathrm{D} w_{l}-\frac{f_{41}(0)}{F G_{c}}\left(C_{l-1}-C_{l+1}\right)-\mathrm{i} \frac{f_{42}(0)}{F G_{c}}\left(C_{l-1}+C_{l+1}\right)=0,
\end{gathered}
$$




$$
\zeta_{l}-\frac{f_{31}(0)}{F G_{c}}\left(C_{l-1}-C_{l+1}\right)-\mathrm{i} \frac{f_{32}(0)}{F G_{c}}\left(C_{l-1}+C_{l+1}\right)=0 .
$$

The boundary conditions at $z \rightarrow \infty$ become

$$
C_{l} \rightarrow 0, \quad w_{l} \rightarrow 0, \quad \mathrm{D} w_{l} \rightarrow 0, \quad \zeta_{l} \rightarrow 0 .
$$

The coefficients $f_{m n}, m=1,2,3,4$ and $n=1,2$, are functions of the basic-state velocity, and given in the Appendix. Note that because the maximum value of the index $l$ needs to be taken as a finite value $L_{n}$, (4.12) is a set of simultaneous ordinary differential equations of order $16 L_{n}+8$ in the computations. Equation (4.12) along with the boundary conditions (4.13) and (4.14) constitute a complex-eigenvalue problem, which can be solved by the shooting technique (Chung \& Chen 2000).

\section{Stability characteristics under inclined rotation}

There are five physical mechanisms affecting the stability of the present system. First, the reduction of buoyancy in the $z$-direction (i.e. the direction of the basic density gradient) due to inclination is a stabilizing factor (Chung \& Chen 2000). Secondly, the gravity (buoyancy) component in the $(x, y)$-plane (parallel to the melt/solid interface) due to inclination is destabilizing. Thirdly, the rotation component in the $z$-direction contributed by spin and/or precession corresponding to the Coriolis force parallel to the $(x, y)$-plane is stabilizing. The fourth is the induced helical flow and the last is the precession component in the $(x, y)$-plane. It was shown by Kropp \& Busse (1991), Busse \& Kropp (1992) and Matthews \& Cox (1997) that an imposed shear flow or a rotation vector on the $(x, y)$-plane is stabilizing when they act individually. However, when acting simultaneously, they may together play a stabilizing or a destabilizing role depending on their relative orientation and amplitude ratio. Similarly, the interaction between basic flow and precession can also apply to the present system. In addition, we note that both the buoyancy reduction in the $z$-direction and the rotation component in the $z$-direction have a 'symmetric action', affecting all instability modes equally in different directions. The other three mechanisms, however, may or may not act symmetrically depending on whether spin is imposed. More precisely, when the system rotates with precession only, these three mechanisms have stationary-oriented components on the $(x, y)$-plane, which consequently destroy the stability symmetry in the $(x, y)$-plane. But nevertheless, when the system rotates with spin with/without precession, these three mechanisms change direction periodically with the same frequency of spin and thus all instability modes travelling in different directions can feel their effects periodically. In this instance the stability symmetry in the $(x, y)$-plane holds.

We examine the stability characteristics of the system under four different kinds of rotation: (i) vertical, (ii) inclined precession, (iii) inclined spin, and (iv) inclined spin and precession. These four cases are influenced by either part or all of the five stability mechanisms discussed above. The physical values considered are shown in table 1, corresponding to lead-tin alloy (Coriell et al. 1980).

\subsection{System rotating vertically}

Since the system rotates vertically, there is no induced basic flow (Lu \& Chen 1997) so that the number of physical parameters involved is much reduced. Specifically, in (4.6) and (4.7), since $S_{n}=0$ and $u_{b}=v_{b}=0$, the linear perturbation equations are 
(a)

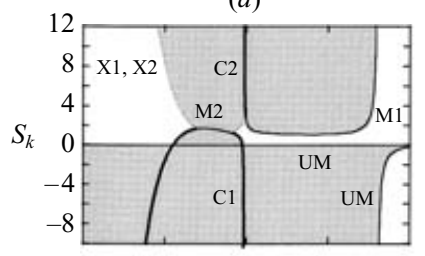

(b)

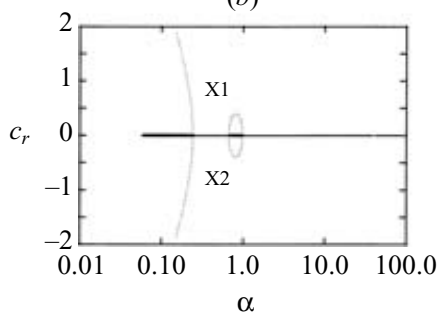

(c)

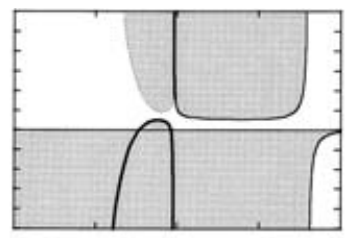

(d)

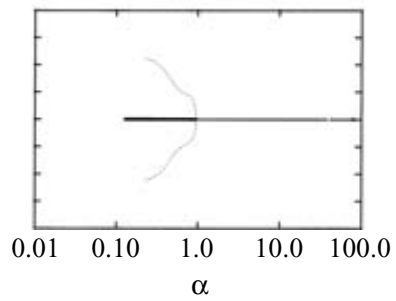

(e)

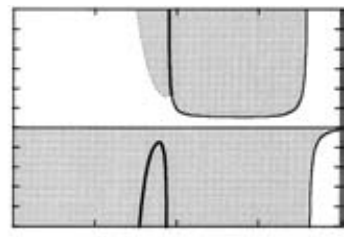

$(f)$

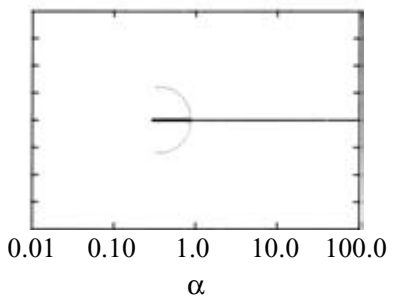

FIGURE 4. The neutral curves in terms of the Sekerka number $S_{k}$ and the wavenumber $\alpha$ and the wave speeds $c_{r}$ of different instability modes for the case of vertical rotation $\left(\phi_{n}=0\right)$. The grey shading shows the unstable region. The labels UM, M1, M2, C1, C2, X1 and X2 denote modes of different stability characteristics. $(a, b) T_{a}=0,(c, d) T_{a}=1,(e, f) T_{a}=4$.

thus independent of $\phi_{\alpha}$ so that the symmetry in the $(x, y)$-plane holds. In addition, the spin Taylor number $T_{a s}$ and the precession Taylor number $T_{a p}$ can be combined into a single Taylor number $T_{a}$.

The numerical results are shown in figure 4, illustrating three cases with different Taylor numbers: $T_{a}=0,1$ and 4. Figures $4(a), 4(c)$ and 4(e) show the neutral curves of the morphological modes M1, M2 and UM (thin curves), the convective modes $\mathrm{C} 1$ and $\mathrm{C} 2$ (thick curves) and the mixed modes X1 and X2 (dotted curves); figures 4(b), $4(d)$ and $4(f)$ show the corresponding wave speeds $c_{r}=-\omega_{i} / \alpha$. In this figure and the subsequent ones, for convenience we have adopted the same labels as used by Forth \& Wheeler (1992) to denote the instability modes and grey shading to denote the unstable region. To help find the physical meaning of these figures, we discuss first the case of $\alpha=0.5$ and $S_{k}=5$ in figure $4(a)$, which is morphologically unstable to mode M2. When $S_{k}$ decreases to a value below the neutral curve of mode M2, the system becomes unstable to mode $\mathrm{C} 1$. For a higher wavenumber, for example $\alpha=10$ and $S_{k}=5$, the system is unstable to mode M1. When the value of $S_{k}$ decreases below the critical value, while remaining positive, the system becomes stable. If the value of $S_{k}$ continues to decrease to zero and becomes negative, then the system becomes morphologically unstable to mode UM. Note that the neutral curves of modes X1, X2 join M1 to form C2, and join C1 to form M2. Moreover, the coalescence of the neutral curves makes the wave speeds of modes X1 and X2 separate into two distinct branches: the wave speed of the left branch is generally larger than that of the right branch (see the dotted curves of figure $4(b)$.

Figure 4(a), similar to figure 7 of Forth \& Wheeler (1992), corresponds to a case without rotation, in which the interaction between the convective mode and morphological mode dominates the system. The neutral curves exhibit a so-called folding structure for the X1 and X2 modes: they have the same stability criteria but travel in opposite directions, i.e. X1 moves forward with wave speed $+c_{r}$ and X2 

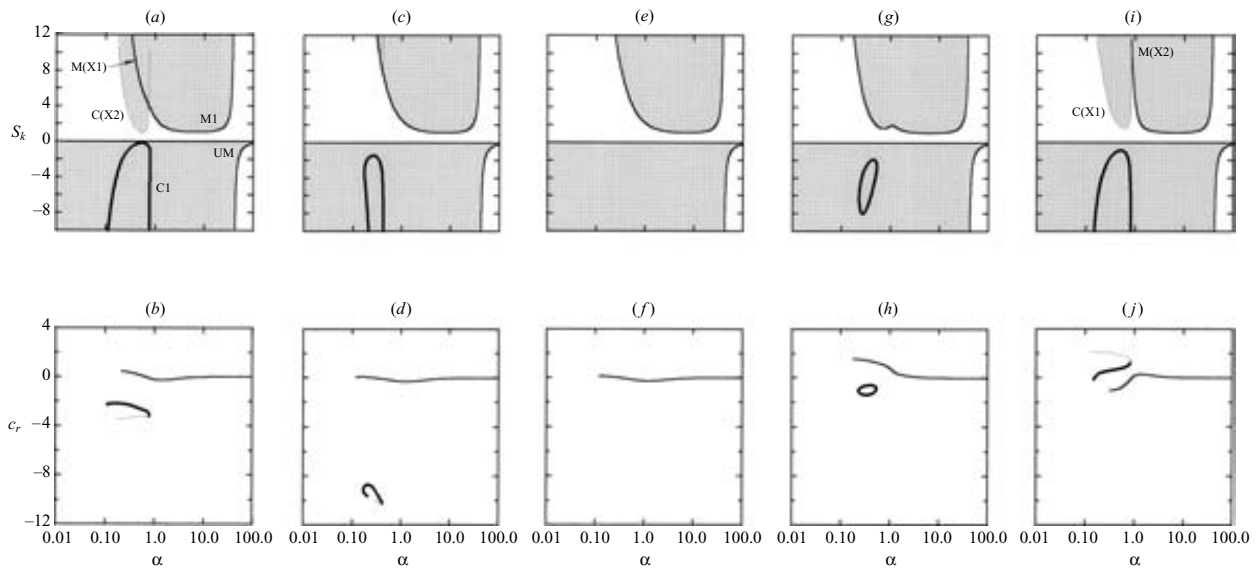

Figure 5. The neutral curves in terms of the Sekerka number $S_{k}$ and the wavenumber $\alpha$ and the wave speeds $c_{r}$ of different stability modes for the case of inclined precession for various propagating angle $\phi_{\alpha}$, where $\phi_{n}=10^{\circ}$ and $T_{a p}=1 .(a, b) \phi_{\alpha}=90^{\circ},(c, d) \phi_{\alpha}=50^{\circ}$, $(e, f) \phi_{\alpha}=0^{\circ},(g, h) \phi_{\alpha}=-74^{\circ},(i, j) \phi_{\alpha}=-80^{\circ}$.

moves backward with wave speed $-c_{r}$. The neutral curves of the stationary modes $\mathrm{C} 1$ and $\mathrm{M} 1$ coalesce with those of X1 and X2, forming two new stationary modes M2 and C2. The UM mode is also stationary but is physically unrealistic because it occurs in the region of non-positive Sekerka number. According to Forth \& Wheeler (1992), the C1 mode is characterized by the flow rising from the troughs of the deformed interface and descending towards the peaks, whereas the M1 mode circulates in the opposite sense. Owing to the interaction between these two modes, the buoyancy-driven rising plume is shifted laterally between the trough and the peak and the interface tends to freeze on one side while dissolving on the other side of the trough, leading to the formation of the travelling modes X1, X2.

We consider two cases when vertical rotation is applied: $T_{a}=1$ and $T_{a}=4$ which are equivalent to rotation speeds of 0.5 r.p.m. (figure $4 c, d$ ) and 1 r.p.m. (figures $4 e, f$ ) respectively. Results show that, due to vertical rotation, the neutral curves of modes $\mathrm{C} 1$ and $\mathrm{X} 1, \mathrm{X} 2$ become disconnected and M2 disappears. This is because modes $\mathrm{C} 1$ and $\mathrm{X} 1, \mathrm{X} 2$ are stabilized by vertical rotation through the action of the Coriolis force, as is commonly found in the similar system of buoyancy-driven convection (for example $\mathrm{Lu} \& \mathrm{Chen}$ 1997). The M1 mode is virtually unaffected due to its short characteristic wavelength compared to the convective modes (Forth \& Wheeler 1992). This implies a situation where M1 will eventually dominate $\mathrm{C} 1$ once the vertical rotation speed becomes large enough, as can be seen in figure 4(e). The wave speeds of X1 and X2 also decrease with increasing rotation speed due to the stabilization by the Coriolis force.

\subsection{System rotating by inclined precession}

As mentioned in $\S 4.1$, when the system rotates with inclined precession the stability is orientation-dependent so that it is necessary to consider $-90^{\circ} \leqslant \phi_{\alpha} \leqslant 90^{\circ}$ for a complete analysis. It is impractical to consider many values of $\phi_{\alpha}$ because of the large computational effort required. We therefore choose the five representative values $\phi_{\alpha}=90^{\circ}, 50^{\circ}, 0^{\circ},-74^{\circ}$ and $-80^{\circ}$ for illustration. Results are shown on figure 5 where 
the tilt angle and the precession speed are fixed respectively at $\phi_{n}=10^{\circ}$ and $T_{a p}=1$ (equivalent to 0.5 r.p.m.). In this case the buoyancy reduction and the precession component in the $z$-direction have the same action on disturbances travelling in different directions in the $(x, y)$-plane. In contrast, the gravity and the precession components act in the $y$-direction only and the basic flow changes direction with height from $220^{\circ}$ (measured with respective to the $x$-axis) at the interface to $180^{\circ}$ in the far field, both breaking the stability symmetry in the $(x, y)$-plane.

By comparing figure 5 with figure 4 , two stability effects due to inclined precession are noticed. First, all the instability modes are oscillatory. Second, the neutral curve of mode X1 (originally travelling forward with speed $+c_{r}$ ) is now smoothly connected onto M1 to form $\mathrm{M}(\mathrm{X} 1)$ and that of mode X2 (originally travelling backward with speed $-c_{r}$ ) is smoothly connected onto $\mathrm{C} 1$ to form $\mathrm{C}(\mathrm{X} 2)$. Similar phenomena can be found in Forth \& Wheeler (1992), who investigated the influence of a shear flow imposed in the $x$-direction on the coupled convective and morphological instabilities of a binary alloy. In the present system, like the imposed shear flow considered by Forth \& Wheeler, the simultaneous presence of the gravity and precession components in the $y$-direction and the induced spiral flow has two similar effects: first, it induces overstability in the modes; secondly, it destroys both the stability symmetry in the $(x, y)$-plane and the folding structure between the $\mathrm{X} 1$ and $\mathrm{X} 2$ modes.

From the wave speeds in figures $5(h)$ and $5(j)$, one may infer that there is likely to exist a marginal angle corresponding to a zero wave speed at which the forward travelling mode $M(X 1)$ switches to the backward travelling mode $M(X 2)$ and the backward travelling modes $\mathrm{M} 1, \mathrm{C} 1$ and $\mathrm{C}(\mathrm{X} 2)$ switch to the forward travelling modes $\mathrm{M} 1, \mathrm{C} 1$ and $\mathrm{C}(\mathrm{X} 1)$, respectively. The marginal angle in the present case is possibly located between $\phi_{\alpha}=-74^{\circ}$ and $\phi_{\alpha}=-80^{\circ}$, determined by the combination of the three asymmetry-driving mechanisms, i.e. the induced flow, the precession and the gravity. A similar result for the marginal angle was obtained by Forth \& Wheeler (1992), in which the marginal direction was perpendicular to the imposed shear flow because the instabilities propagating in this direction could not 'sense' the imposed flow.

Regarding the stability criterion, it is seen that the critical Sekerka number of mode M1 is virtually independent of the propagating angle $\phi_{\alpha}$. In contrast, the criteria for modes $\mathrm{C} 1, \mathrm{C}(\mathrm{X} 1)$ and $\mathrm{C}(\mathrm{X} 2)$ are quite sensitive to the variation of $\phi_{\alpha}$, namely both the convective modes $\mathrm{C} 1$ and $\mathrm{C}(\mathrm{X} 2)$ are most unstable in the direction $\phi_{\alpha}=90^{\circ}$ (parallel with the $y$-axis) as shown in figure $5(a)$, and are largely suppressed along $\phi_{\alpha}=0^{\circ}$ (parallel with the $x$-axis) as shown in figure $5(e)$. This is because, due to inclined precession, both gravity and precession act in the $y$-direction only, so that they have no effect on the modes travelling in the $x$-direction, whereas the modes travelling in the $x$-direction are stabilized by the other three factors - the buoyancy reduction in the $z$-direction, the rotation vector in the $z$-direction and the induced basic flow. Note that for the case in figure 5(e) the basic flow plays a stabilizing role for the modes travelling in the $x$-direction because the modes cannot 'sense' the precession component, which in this instance is acting in the $y$-direction. For other modes propagating in directions other than $\phi_{\alpha}=0^{\circ}$, it is inferred that the interaction between the basic flow and the precession component in the $y$-direction is destabilizing. This inference is made based on the work of Matthews \& Cox (1997), who examined buoyancy-driven convection under the interaction of a horizontal rotation vector and an imposed shear flow, finding that the imposed shear and rotation may together play a destabilizing role. We will discuss the application of their work in more detail in the case involving both precession and spin. 
(a)

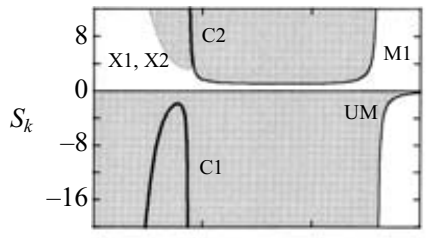

(b)

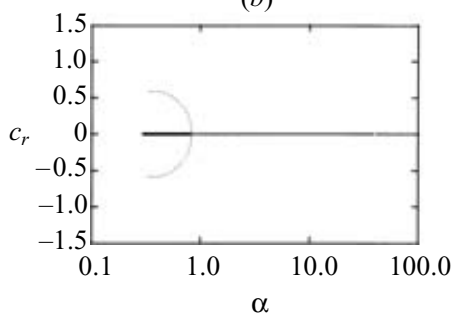

(c)

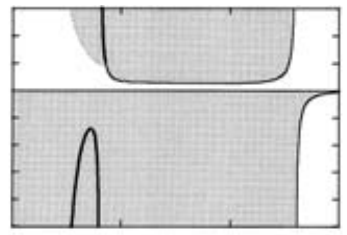

(d)

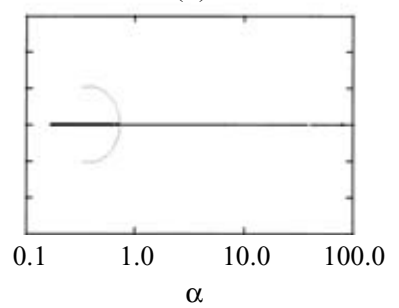

(e)

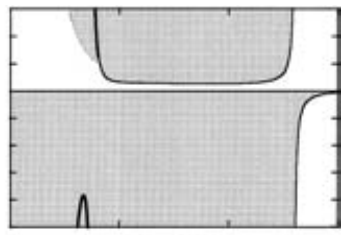

$(f)$

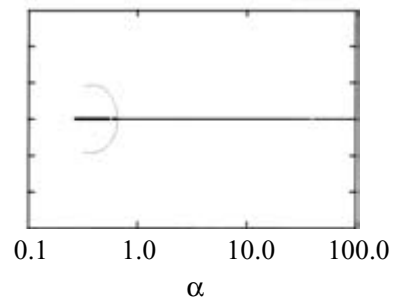

Figure 6. The neutral curves in terms of the Sekerka number $S_{k}$ and the wavenumber $\alpha$ and the wave speeds $c_{r}$ of different stability modes for the case of inclined spin for various inclination angles $\phi_{n}$, where $\Omega=80$ and $T_{a p}=0 .(a, b) \phi_{n}=10^{\circ},(c, d) \phi_{n}=20^{\circ},(e, f)$ $\phi_{n}=25^{\circ}$.

\subsection{System rotating by inclined spin}

In this case, the precession component in the $(x, y)$-plane is not active but the other four mechanisms are. As in the previous cases, the buoyancy reduction in the $z$-direction and the rotation component in the $z$-direction (equal to the spin vector for the present case) will not interfere with the stability symmetry in the $(x, y)$-plane. Moreover, because of spin, both the induced flow and the gravity component in the $(x, y)$-plane change direction synchronously with the spin, namely the induced flow and gravity component also rotate with the spin frequency $\Omega$. It follows that instability modes travelling in different directions will have an equal stability condition and the system will retain the stability symmetry in the $(x, y)$-plane. Note also that the basic flow velocity increases with increasing inclination angle and decreases with increasing spin frequency $\Omega$. Bearing these features in mind, we examine the system's stability under the effects of inclined spin.

We show in figure 6 the neutral curves for the case of $\Omega=80$ (equivalent to $T_{a s}^{1 / 2} \approx 2$ or 1 r.p.m. for the present system) with the inclination angle $\phi_{n}$ varying from $10^{\circ}$ to $25^{\circ}$. A major result of inclined spin is seen by comparing figure $6(a)$ with figure $4(a)$ : the mixed modes X1, X2 and the convective modes C1, C2 are largely stabilized while the morphological mode M1 is slightly stabilized. When the inclination angle increases (figure $6 c, e$ ), the stabilization due to inclined spin is enhanced. By comparing figure 6(a) with figure $5(a)$, we examine the difference between inclined spin and inclined precession and find that the mixed modes X1, X2 and convective mode C2 absent in the inclined precession case appear in the spin case. Both the folding structure of the $\mathrm{X} 1$ and $\mathrm{X} 2$ modes and the stability symmetry in the $(x, y)$-plane destroyed in the inclined precession case also reappear here. Note that $\mathrm{C} 1, \mathrm{C} 2, \mathrm{M} 1$ and UM have $c_{r}=0$ (see figure $6 b, d$ and $f$ ), indicating that these modes move synchronously with the motion of spin. On the other hand, X1 and X2 have $c_{r} \neq 0$, indicating that the mixed modes move non-synchronously with spin and their frequencies are modulated by $\omega_{i}=-\alpha c_{r}$ (see equation (4.11)). 

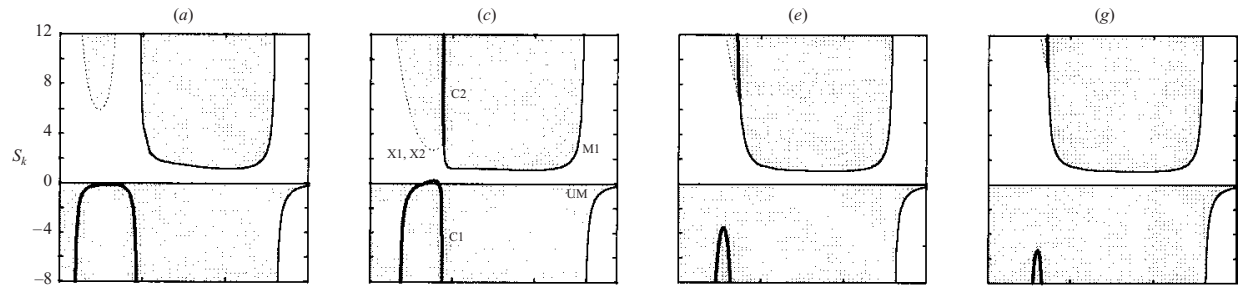

(b)

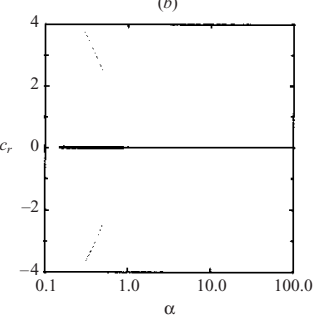

(d)

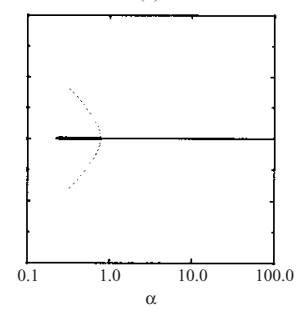

$(f)$

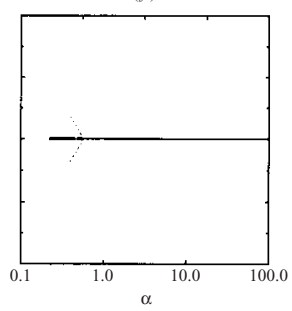

(h)

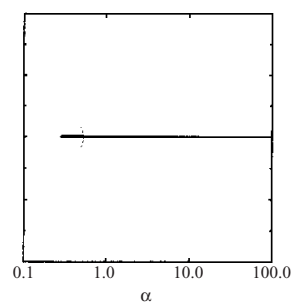

FIgURE 7. The neutral curves in terms of the Sekerka number $S_{k}$ and the wavenumber $\alpha$ and the wave speeds $c_{r}$ of different stability modes for the case of inclined spin with precession for various precession parameter $(-1)^{n_{p}} T_{a p}^{1 / 2}$, where $\phi_{n}=20^{\circ}$ and $\Omega=80 .(a, b)(-1)^{n_{p}} T_{a p}^{1 / 2}=-1$, $(c, d)(-1)^{n_{p}} T_{a p}^{1 / 2}=-0.8,(e, f)(-1)^{n_{p}} T_{a p}^{1 / 2}=0.5,(g, h)(-1)^{n_{p}} T_{a p}^{1 / 2}=1$.

\subsection{System rotating by inclined spin and precession}

We examine the stability characteristics for the case $\Omega=80$ (equivalent to $T_{a s}^{1 / 2} \approx 2$ ) and $\phi_{n}=20^{\circ}$ for various values of $(-1)^{n_{p}} T_{a p}^{1 / 2}$ and the results are shown on figure 7 . In this case, all five stability mechanisms are active and because of spin they influence equally all instability modes travelling in different directions. Consequently, the stability symmetry in the $(x, y)$-plane and the folding structure of the mixed modes are retained. Moreover, modes M1, C1 and C2 move synchronously with spin while X1 and X2 move non-synchronously. It is shown in figure 7 that the stability criterion of mode M1 remains virtually the same for different $T_{a p}$. The most-unstable mode is $\mathrm{C} 1$ occurring at $(-1)^{n_{p}} T_{a p}^{1 / 2}=-0.8$ (figure $7 c$ ) and modes $\mathrm{C} 1, \mathrm{C} 2, \mathrm{X} 1$ and X2 are significantly suppressed at other values of $T_{a p}$, for example at $(-1)^{n_{p}} T_{a p}^{1 / 2}=-1$ (figure $7 a$ ), $(-1)^{n_{p}} T_{a p}^{1 / 2}=0.5$ (figure $7 e$ ) and $(-1)^{n_{p}} T_{a p}^{1 / 2}=1$ (figure $7 g$ ). The comparison of the neutral curves of the $\mathrm{C} 1$ mode in figures $7(c), 7(e)$ and $7(\mathrm{~g})$ indicates that $\mathrm{C} 1$ becomes unstable at larger negative values of $(-1)^{n_{p}} T_{a p}^{1 / 2}$ because the stabilizing action due to the rotation component in the $z$-direction (in terms of $T_{a z}=C_{n}(-1)^{n_{p}} T_{a p}^{1 / 2}+T_{a s}^{1 / 2}$ ) becomes smaller at larger negative values of $(-1)^{n_{p}} T_{a p}^{1 / 2}$. However, this fails to explain why the $\mathrm{C} 1$ mode in figure $7(a)$ is more stable than that of figure $7(c)$ despite $(-1)^{n_{p}} T_{a p}^{1 / 2}=-1$ in figure $7(a)$ being even more negative. A plausible explanation for this can be obtained from the interaction between the basic flow and the precession component in the $(x, y)$-plane, which is discussed in more detail below.

We summarize in figure 8 the stability characteristics in terms of the relationship between $S_{k}^{c}$ and $(-1)^{n_{p}} T_{a p}^{1 / 2}$. The stable region, marked by the grey shading, is enclosed by the critical stability curves of the M1, C1 and UM modes. The stability criterion of the M1 mode is virtually unchanged on varying $(-1)^{n_{p}} T_{a p}^{1 / 2}$. However, the $\mathrm{C} 1$ mode is significantly stabilized when $(-1)^{n_{p}} T_{a p}^{1 / 2}>-0.5$ but is slightly destabilized when $(-1)^{n_{p}} T_{a p}^{1 / 2}<-0.5$ except in a small region near $(-1)^{n_{p}} T_{a p}^{1 / 2}=-1$ where the $\mathrm{C} 1$ mode 


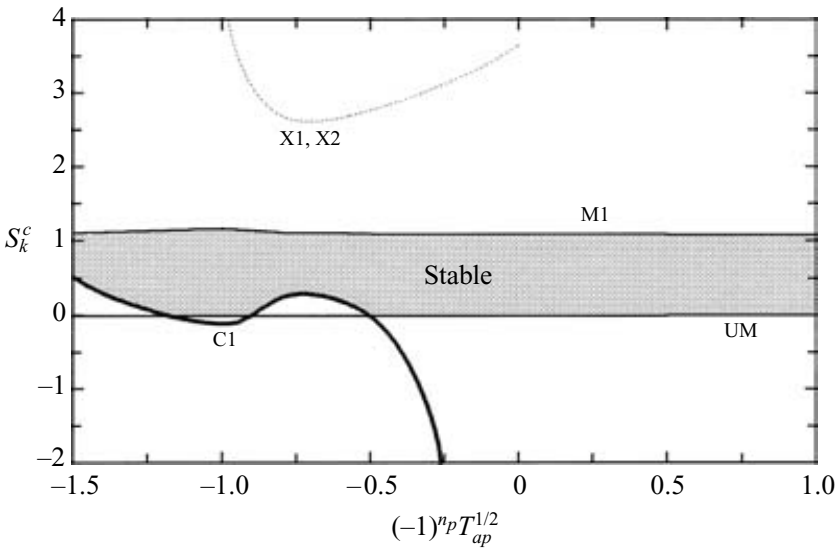

FiguRE 8. The critical Sekerka number $S_{k}^{c}$ versus the precession parameter $(-1)^{n_{p}} T_{a p}^{1 / 2}$ for the cases of figure 7 . The grey area represents the stable region for all instability modes.

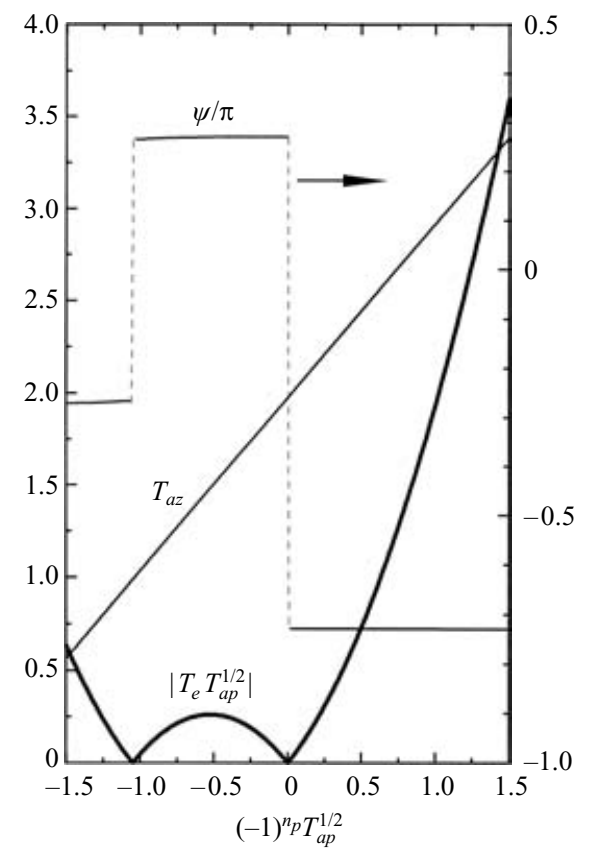

FIGURE 9. The parameters $\left|T_{e} T_{a p}^{1 / 2}\right|, T_{a z}$ and $\psi$ versus the precession parameter $(-1)^{n_{p}} T_{a p}^{1 / 2}$ for the cases of figure 7. The parameter $\left|T_{e} T_{a p}^{1 / 2}\right|$ is the amplitude ratio between precession and the induced basic flow, $T_{a z}=C_{n}(-1)^{n_{p}} T_{a p}^{1 / 2}+T_{a s}^{1 / 2}$ is the rotation component along the height of the system, and $\psi$ is the angle between the direction of the precession component in the $(x, y)$-plane and the induced flow at melt/solid interface.

is also stabilized. To interpret the stabilization of mode $\mathrm{C} 1$ in this small region, we illustrated in figure 9 the variations of $T_{a z}, \psi$ and $\left|T_{e} T_{a p}^{1 / 2}\right|$ with $(-1)^{n_{p}} T_{a p}^{1 / 2}$, where $\psi$ is the relative direction (in radians) of the basic flow measured with respect to the precession component in the $(x, y)$-plane.

Although the direction of the basic flow changes with height in the Ekman layer, it is reasonable to choose the representative value of $\psi$ at the melt/solid interface 
because the convective mode is largely confined in the solute boundary layer near the interface. The parameter $\left|T_{e} T_{a p}^{1 / 2}\right|$ is the ratio of the amplitude of the precession component in the $(x, y)$-plane to that of the basic flow, which is obtained by (3.12) in the limits of large Schmidt number $S_{c}$ and large Lewis number $L_{e}$. The results of figure 9 show that the rotation component in the $z$-direction given by $T_{a z}$ decreases with decreasing $(-1)^{n_{p}} T_{a p}^{1 / 2}$, indicating that the stabilizing effect of $T_{a z}$ gradually diminishes as $(-1)^{n_{p}} T_{a p}^{1 / 2}$ decreases. This explains why the convective $\mathrm{C} 1$ mode becomes unstable as $(-1)^{n_{p}} T_{a p}^{1 / 2}$ decreases from figure $7(g)$ through $7(e)$ to $7(c)$. Note that in figure 9 there are two zeros of $\left|T_{e} T_{a p}^{1 / 2}\right|$, one at $(-1)^{n_{p}} T_{a p}^{1 / 2}=0$ and the other close to $(-1)^{n_{p}} T_{a p}^{1 / 2}=-1$, implying that the amplitude ratio between the basic flow and the precession component is quite large near these two points. Matthews \& Cox (1997) investigated buoyancy-driven convection under the interaction between an imposed shear flow and a rotation vector lying in the plane parallel to the shear flow. They found that the convective instability tends to be suppressed when the following two conditions both hold: (1) the shear flow is relatively strong compared to the rotation and (2) these two mechanisms have virtually the same direction of vorticity (see figure 5 of Matthews \& Cox 1997); Applying their findings to our case, to have the same direction of vorticity the relative orientation measured from the precession component in the $(x, y)$-plane to the basic flow needs to be close to $90^{\circ}$. Because the relative orientation in the region $-1 \leqslant(-1)^{n_{p}} T_{a p}^{1 / 2}<0$ shown in figure 9 is about $\psi \approx 60^{\circ}$ and the basic flow is relatively strong compared to the precession component near $(-1)^{n_{p}} T_{a p}^{1 / 2}=-1$ and 0 where $\left|T_{e} T_{a p}^{1 / 2}\right| \rightarrow 0$, we infer that the interaction between the basic flow and the precession component in the $(\mathrm{x}, \mathrm{y})$-plane is stabilizing to mode $\mathrm{C} 1$ near these two points. This then is the reason why the $\mathrm{C} 1$ mode becomes more stable in figure $7(a)$ where $(-1)^{n_{p}} T_{a p}^{1 / 2}=-1$ than in figure $7(c)$ where $(-1)^{n_{p}} T_{a p}^{1 / 2}=-0.8$. For morenegative values of $(-1)^{n_{p}} T_{a p}^{1 / 2}$ than -1 , as shown in figure 9 the relative orientation becomes $\psi \approx-45^{\circ}$, indicating that the precession component in the $(x, y)$-plane and the shear flow have virtually oppositely directed vorticity and so the interaction between them becomes destabilizing, making mode $\mathrm{C} 1$ become unstable again.

To further elucidate this scenario, we illustrate another example by comparing figure 7(e) to figure $6(c)$. Both cases have $\Omega=80$ and $\phi_{n}=20^{\circ}$, while figure $6(c)$ has $(-1)^{n_{p}} T_{a p}^{1 / 2}=0$ and figure $7(e)$ has $(-1)^{n_{p}} T_{a p}^{1 / 2}=0.5$, namely both cases have the same intensity of buoyancy reduction in the $z$-direction and the same gravity component in the $(x, y)$-plane but the stabilizing rotation vector in the $z$-direction in the inclinedspin case (figure $6 c$ ) is weaker than that in the inclined-spin-with-precession case (figure 7e). The comparison shows that the critical value of the $\mathrm{C} 1$ mode in figure 6(c) is about $S_{k}=-6$ and in figure $7(e)$ about $S_{k}=-3$, indicating surprisingly that C1 is more stabilized in figure $6(c)$ although the rotation vector in the $z$-direction is weaker there. This result also can be explained by taking into account the interaction between the basic flow and the precession component in the $(x, y)$-plane. For the inclined-spin case in figure $6(c)$, there is no precession applied, i.e. $(-1)^{n_{p}} T_{a p}^{1 / 2}=0$, so the basic flow is itself a stabilizing factor. In contrast, for the inclined-spin-withprecession case of figure $7(e)$, the value $(-1)^{n_{p}} T_{a p}^{1 / 2}=0.5$ corresponds to $\psi \approx-130^{\circ}$ as shown in figure 9, implying that the precession component and the basic flow have virtually oppositely-directed vorticity and they together play a destabilizing role. In words, the destabilizing action due to the collaboration of the precession component in the $(x, y)$-plane and the basic flow has prevailed over the stabilizing action by the rotation vector in the $z$-direction for the case in figure $7(e)$, rendering mode $\mathrm{C} 1$ more stabilized in figure $6(c)$. 


\section{Conclusions}

We have analysed the stability characteristics of a directionally solidifying binary alloy under inclined rotation. Before the onset of instability occurs, the basic state is mainly a strong helical shear flow induced by the inclination and modified by the rotation, moving along the melt/solid interface. The corresponding basic-state temperature and concentration remain the same as those where the system is vertical with/without rotation. The induced helical flow, which increases in magnitude with increasing inclination angle and decreases with increasing rotation speed, consists of three components: the solutal-layer flow, the thermal-layer flow and the Ekman-layer flow. For a lead-tin alloy with large Lewis number, the thermal-layer flow ultimately dominates the velocity away from the melt/solid interface. The solutal-layer flow is confined in the shallow solute layer above the interface, decaying from the interface with the length scale of $D_{f} / V$, and the Ekman-layer flow is confined in the Ekman layer above the interface with depth virtually inversely proportional to $T_{e}$. Relative to the solid, the basic flow is steady when only inclined precession is applied, while it changes direction periodically with the spin frequency once inclined spin is imposed.

The linear stability analyses shows that in the present system there are five mechanisms collaborating or competing to influence the stability of the system. They are:

(i) the reduction of buoyancy along the height of the system (i.e. along the direction of the basic density gradient) due to inclination;

(ii) the rotation vector along the height of the system due to either spin or precession, which corresponds to the Coriolis force acting parallel to the $(x, y)$-plane;

(iii) the gravity (buoyancy) component in the $(x, y)$-plane due to inclination;

(iv) the rotation component in the $(x, y)$-plane, which can be generated only by inclined precession;

(v) the spiral basic-state flow induced by inclination and modified by rotation. Of these five driving mechanisms, the first and second are stabilizing and the third is destabilizing; the last two play a stabilizing or destabilizing role, depending on their relative orientation and amplitude ratio. The first and second mechanisms will not interfere with the stability symmetry in the $(x, y)$-plane or the folding structure of the mixed modes, whereas the other three mechanisms may or may not destroy the stability symmetry in the $(x, y)$-plane and the folding structure of the mixed modes depending on whether spin is imposed. When the system rotates with inclined spin with/without precession, these three mechanisms change direction synchronously with the motion of spin. Therefore the instability modes travelling in different directions can 'sense' these three mechanisms periodically, and the stability symmetry in the $(x, y)$-plane and the folding structure for the mixed modes are accordingly retained. On the other hand, when the system rotates with precession only, these three mechanisms act with stationary orientations and destroy both the stability symmetry in the $(x, y)$-plane and the folding structure of the mixed modes.

When the system remains vertical, with or without rotation, the morphological and convective modes are stationary and the mixed modes are oscillatory. When only inclined-precession is imposed, all instability modes become oscillatory. When inclinedspin-with/without-precession is applied, all instability modes are also oscillatory, the morphological and convective modes move synchronously with spin while the mixed modes moves non-synchronously with spin. Regarding the stability criterion, the morphological instability is slightly stabilized by inclined rotation while the mixed mode and convective mode are significantly stabilized. Because inclined precession may destroy the symmetry in the $(x, y)$-plane and become destabilizing 
when interacting with the basic shear flow, we suggest that, in the industrial application of preventing the final castings from compositional non-uniformities, inclined spin is more effective than inclined precession.

Finally, a further remark regarding the comparison between our earlier work (Chung \& Chen 2000) and the present work is in order. As stated in the introductory section, both these works consider the effects of inclined spin and precession, while in the earlier work the discussion was focused on the buoyancy-driven instability in the mush and in the present study the investigation is devoted to the morphological and convective instability in the melt. In the earlier work, although the five stability mechanisms also exist, the most significant instability mechanisms in the mush are the reduction of buoyancy along the height and the induced basic flow, both resulting in a more stable state of the mush. The other mechanisms are weak because of the large resistance to the flow in the dendrite mush, so that the effects of the Coriolis force are negligible and the flow in the mush becomes monotonically more stable with increasing inclination angle. In addition, the breakdown of the stability symmetry in the $(x, y)$-plane for the mush is primarily caused by the basic flow, which moves in the direction parallel to the gravity component in the $(x, y)$-plane. As a result, the most-unstable mode propagates in the direction perpendicular to the basic flow. In the present system, however, the Coriolis force is much stronger in the melt, which then interacts with the basic blow and causes more complex stability results, as presented in the previous section.

We would like to thank the National Science Council of Taiwan for financial support through Grant No. NSC 90-2212-E-002-202.

\section{Appendix. The coefficients of the perturbation equations (4.12) and (4.13)}

$$
\begin{aligned}
f_{11}(z)= & A_{c}\left[\mathrm{e}^{-z / d_{E}} \cos \left(b z-\phi_{c}\right)-\mathrm{e}^{-z} \cos \left(\phi_{c}\right)\right]+A_{t}\left[\mathrm{e}^{-z / d_{E}} \cos \left(b z-\phi_{t}\right)-\mathrm{e}^{-z / L_{e}} \cos \left(\phi_{t}\right)\right], \\
f_{12}(z)= & A_{c}\left[\mathrm{e}^{-z / d_{E}} \sin \left(b z-\phi_{c}\right)+\mathrm{e}^{-z} \sin \left(\phi_{c}\right)\right]+A_{t}\left[\mathrm{e}^{-z / d_{E}} \sin \left(b z-\phi_{t}\right)+e^{-z / L_{e}} \sin \left(\phi_{t}\right)\right], \\
f_{21}(z)= & A_{c}\left[-\left(\frac{1}{d_{E}^{2}}-b^{2}\right) \mathrm{e}^{-z / d_{E}} \cos \left(b z-\phi_{c}\right)+\mathrm{e}^{-z} \cos \left(\phi_{c}\right)-\frac{2 b}{d_{E}} \mathrm{e}^{-z / d_{E}} \sin \left(b z-\phi_{c}\right)\right] \\
& +A_{t}\left[-\left(\frac{1}{d_{E}^{2}}-b^{2}\right) \mathrm{e}^{-z / d_{E}} \cos \left(b z-\phi_{t}\right)+\frac{\mathrm{e}^{-z / L_{e}}}{L_{e}^{2}} \cos \left(\phi_{t}\right)-\frac{2 b}{d_{E}} \mathrm{e}^{-z / d_{E}} \sin \left(b z-\phi_{t}\right)\right], \\
f_{22}(z)= & A_{c}\left[-\left(\frac{1}{d_{E}^{2}}-b^{2}\right) \mathrm{e}^{-z / d_{E}} \sin \left(b z-\phi_{c}\right)-\mathrm{e}^{-z} \sin \left(\phi_{c}\right)+\frac{2 b}{d_{E}} \mathrm{e}^{-z / d_{E}} \cos \left(b z-\phi_{c}\right)\right] \\
& +A_{t}\left[-\left(\frac{1}{d_{E}^{2}}-b^{2}\right) \mathrm{e}^{-z / d_{E}} \sin \left(b z-\phi_{t}\right)-\frac{\mathrm{e}^{-z / L_{e}}}{L_{e}^{2}} \sin \left(\phi_{t}\right)-\frac{2 b}{d_{E}} \mathrm{e}^{-z / d_{E}} \cos \left(b z-\phi_{t}\right)\right], \\
f_{31}(z)= & A_{c}\left[\frac{\mathrm{e}^{-z / d_{E}}}{d_{E}} \sin \left(b z-\phi_{c}\right)+\mathrm{e}^{-z} \sin \left(\phi_{c}\right)-b \mathrm{e}^{-z / d_{E}} \cos \left(b z-\phi_{c}\right)\right] \\
& +A_{t}\left[\frac{\mathrm{e}^{-z / d_{E}}}{d_{E}} \sin \left(b z-\phi_{t}\right)+\frac{\mathrm{e}^{-z / L_{e}}}{L_{e}} \sin \left(\phi_{t}\right)-b \mathrm{e}^{-z / d_{E}} \cos \left(b z-\phi_{t}\right)\right], \\
& +A_{t}\left[-\frac{\mathrm{e}^{-z / d_{E}}}{d_{E}} \cos \left(b z-\phi_{t}\right)+\frac{\mathrm{e}^{-z / L_{e}}}{L_{e}} \cos \left(\phi_{t}\right)-b \mathrm{e}^{-z / d_{E}} \sin \left(b z-\phi_{t}\right)\right], \\
f_{32}(z)= & A_{c}\left[-\frac{\mathrm{e}^{-z / d_{E}}}{d_{E}} \cos \left(b z-\phi_{c}\right)+\mathrm{e}^{-z} \cos \left(\phi_{c}\right)-b \mathrm{e}^{-z / d_{E}} \sin \left(b z-\phi_{c}\right)\right] \\
& {[-1, b}
\end{aligned}
$$




$$
\begin{aligned}
f_{41}(z)= & A_{c}\left[\frac{\mathrm{e}^{-z / d_{E}}}{d_{E}} \cos \left(b z-\phi_{c}\right)-\mathrm{e}^{-z} \cos \left(\phi_{c}\right)+b \mathrm{e}^{-z / d_{E}} \sin \left(b z-\phi_{c}\right)\right] \\
& +A_{t}\left[\frac{\mathrm{e}^{-z / d_{E}}}{d_{E}} \cos \left(b z-\phi_{t}\right)-\frac{\mathrm{e}^{-z / L_{e}}}{L_{e}} \cos \left(\phi_{t}\right)+b \mathrm{e}^{-z / d_{E}} \sin \left(b z-\phi_{t}\right)\right], \\
f_{42}(z)= & A_{c}\left[\frac{\mathrm{e}^{-z / d_{E}}}{d_{E}} \sin \left(b z-\phi_{c}\right)+\mathrm{e}^{-z} \sin \left(\phi_{c}\right)-b \mathrm{e}^{-z / d_{E}} \cos \left(b z-\phi_{c}\right)\right], \\
& +A_{t}\left[\frac{\mathrm{e}^{-z / d_{E}}}{d_{E}} \sin \left(b z-\phi_{t}\right)+\frac{\mathrm{e}^{-z / L_{e}}}{L_{e}} \sin \left(\phi_{t}\right)-b \mathrm{e}^{-z / d_{E}} \cos \left(b z-\phi_{t}\right)\right] .
\end{aligned}
$$

In the above equations, the coefficients $A_{c}$ and $A_{t}$ are

$$
A_{c}=\alpha S_{n} R_{c} G_{c} /\left(2 \Delta_{c}\right), \quad A_{t}=\alpha S_{n} R_{t} G_{l} L_{e} /\left(2 \Delta_{t}\right) .
$$

\section{REFERENCES}

BrattKus, K. \& Davis, S. H. 1988 Flow induced morphological instabilities: Stagnation-point flows, J. Cryst. Growth 89, 423-427.

Busse, F. H. \& Kropp, M. 1992 Buoyancy driven instabilities in rotating layers with parallel axis of rotation. Z. Angew. Math. Phys. 43, 28-35.

Chen, Y. J. \& DAvis, S. H. 1999 Directional solidification of a binary alloy into a cellular convective flow: localized morphologies. J. Fluid Mech. 395, 253-270.

Chung, C. A. \& Chen, F. 2000 Convection in directionally solidifying alloys under inclined rotation. J. Fluid Mech. 412, 93-123.

Chung, C. A. \& Chen, F. 2001 Morphological instability in a directionally solidifying solution with an imposed shear flow. J. Fluid Mech. 436, 85-106.

Coriell, S. R., Cordes, M. R., Boettinger, W. J. \& Sekerka, R. F. 1980 Convective and interfacial instabilities during unidirectional solidification of a binary alloy. J. Cryst. Growth 49, 13-28.

Coriell, S. R., McFadden G. B., Boisvert, R. F. \& Sekerka, R. F. 1984 Effect of a forced Couette flow on coupled convective and morphological instabilities during unidirectional solidification. J. Cryst. Growth 69, 15-22.

Davalos-Orozco, L. A. \& Busse, F. H. 2002 Instability of a thin film flowing on a rotating horizontal or inclined plane. Phys. Rev. E 65, 026312, 1-10.

Davis, S. H. 1990 Hydrodynamic interaction in directional solidification. J. Fluid Mech. 212, 242-262.

Davis, S. H. \& Schulze, T. P. 1996 Effects of flow on morphological stability during directional solidification. Metall. Mater. Trans. A 27, 583-594.

Delves, R. T. 1968 Theory of stability of a solid-liquid interface during growth from stirred melts. J. Cryst. Growth 3, 562-568.

Delves, R. T. 1971 Theory of the stability of a solid-liquid interface during growth from stirred melts - II. J. Cryst. Growth 8, 13-25.

Forth, S. A. \& WheELer, A. A. 1989 Hydrodynamic and morphological stability of the unidirectional solidification of a freezing binary alloy: a simple model. J. Fluid Mech. 202, $339-366$.

Forth, S. A. \& Wheeler, A. A. 1992 Coupled convective and morphological instability in a simple model of the solidification of a binary alloy, including a shear flow. J. Fluid Mech. 236, 61-94.

Glicksman, M. E., Coriell, S. R. \& McFadden, G. B. 1986 Interaction of flows with the crystal-melt interface. Annu. Rev. Fluid Mech. 18, 307-335.

Hoffmann, N. P. \& Busse, F. H. 2001 Linear instability of Poiseuille-Couette-Ekman flows: Local results for flows between differentially rotating disks with throughflow. Phys. Fluids 13, 2735-2738.

Jenkins, D. R. 1985 Nonlinear analysis of convective and morphological instability during solidification of a dilute binary alloy. IMA J. Appl. Maths 35, 145-157.

Kropp, M. \& Busse, F. H. 1991 Thermal convection in differentially rotating system. Geophys. Astrophys. Fluid Dyn. 61, 127-148. 
Langer, J. S. 1980 Instabilities and pattern formation in crystal growth. Rev. Mod. Phys. 52, 1-28.

LU, J. W. \& Chen, F. 1997 Rotation effects on the convection of binary alloys unidirectionally solidified from below, Intl J. Heat Mass Transfer 40, 237-246.

McFadden, G. B., Coriell, S. R. \& Alexander, J. I. D. 1988 Hydrodynamic and free boundary instabilities during crystal growth: the effect of a plane stagnation flow. Commun. Pure Appl. Maths 41, 683-706.

Matthews, P. \& Cox, S. 1997 Linear stability of rotating convection in an imposed shear flow. J. Fluid Mech. 350, 271-293.

Mullins, W. W. \& SeKerka, R. F. 1964 Stability of a planar interface during solidification of a binary alloy. J. Appl. Phys. 35, 444-451.

Oztekin, A. \& Pearlstein, A. J. 1992 Coriolis effects on the stability of plane-front solidification of dilute $\mathrm{Pb}-\mathrm{Sn}$ binary alloys Metall Trans. 23b, 73-80.

Powell, M. J. 1970 Numerical Methods for Nonlinear Algebraic Equations. Gordon \& Breach.

Riley, D. S. \& Davis S. H. $1990 a$ Long-wave interactions in morphological and convective instabilities. IMA J. Appl. Maths 45, 267-285.

Riley, D. S. \& Davis S. H. $1990 b$ Long-wave morphological instabilities in the directional solidification of a dilute binary mixture. SIAM J. Appl. Maths 50, 420-436.

Rutter, J. W. \& Chalmers, B. A. 1953 A prismatic substructure formed during solidification of metals. Can. J. Phys. 31, 15-39.

Sample, A. K. \& Hellawell, A. 1984 The mechanism of formation and prevention of channel segregation during alloy solidification. Metall. Trans. A 15, 2163-2173.

Schulze, T. P. \& DAVIS, S. H. 1995 Shear stabilization of morphological instability during directional solidification. J. Cryst. Growth 149, 253-265.

UngaR, L. H \& BROWN R. A. 1984 Cellular interface morphologies in directional solidification. The one-sided model. Phys. Rev. B 29, 1367-1380.

Volfson, D. \& Vinals, J. 2001 Morphological stability analysis of directional solidification into an oscillatory fluid layer. Phys. Fluids 13, 3599-3609.

WheELER, A. A. 1991 A strongly nonlinear analysis of the morphological instability of a freezing binary alloy: solutal convection, density change, and nonequilibrium effects. IMA J. Appl. Maths 47, 173-192.

Wollkind, D. J. \& Segel, L. A. 1970 A nonlinear stability analysis of the freezing of a dilute binary alloy. Phil. Trans. R. Soc. Lond. A 268, 351-380. 